\title{
On the Hadamard Formula for Second Order Systems in Non-Smooth Domains
}

\author{
Vladimir Kozlov and Sergey Nazarov
}

\section{Linköping University Post Print}

N.B.: When citing this work, cite the original article.

This is an electronic version of an article published in:

Vladimir Kozlov and Sergey Nazarov, On the Hadamard Formula for Second Order Systems in Non-Smooth Domains, 2012, Communications in Partial Differential Equations, (37), 5, 901-933.

Communications in Partial Differential Equations is available online at informaworld ${ }^{\mathrm{TM}}$ : http://dx.doi.org/10.1080/03605302.2011.626100

Copyright: Taylor \& Francis: STM, Behavioural Science and Public Health Titles / Taylor \& Francis

http://www.tandf.co.uk/journals/default.asp

Postprint available at: Linköping University Electronic Press

http://urn.kb.se/resolve?urn=urn:nbn:se:liu:diva-77549 


\title{
On the Hadamard formula for second order systems in non-smooth domains
}

\author{
Vladimir Kozlov ${ }^{a}$ and Sergey Nazarov ${ }^{b}$ \\ ${ }^{a}$ Department of Mathematics, Linköping University, \\ S-581 83 Linköping, Sweden \\ ${ }^{b}$ Institute for Problems in Mechanical Engineering, \\ Russian Academy of Sciences, \\ V.O., Bol'shoy pr. 61, St Petersburg 199178, RF
}

\begin{abstract}
Perturbations of eigenvalues of the Dirichlet problem for a second order elliptic system in a bounded domain $\Omega$ in $\mathbb{R}^{n}$ are studied under variations of the domain $\Omega$. We investigate the case when the perturbed domain is located in a $d$-neighborhood of the reference Lipschitz domain. A new asymptotic formula is derived; it contains terms that are absent in the classical formula of Hadamard. The latter is valid only for smooth domains and smooth perturbations. We give conditions that guarantee the validity of the Hadamard formula. The general asymptotic formula is applied when $\Omega$ is perturbed by small curvilinear and circular cuts. Most of the results are new even for the Laplace operator.
\end{abstract}

\section{Introduction}

One of the classical shape optimization problems deals with minimization of eigenvalues of a boundary value problem for elliptic operator in a bounded domain. In this problem, the optimization parameter is the domain, possibly subject to certain constraints. A standard approach to such a problem is to apply asymptotic formulae for eigenvalues when the domain is varied, and one of the first examples of such an asymptotic formula is the Hadamard formula for the first eigenvalue of the Dirichlet Laplacian (see [6]):

$$
\lambda\left(h_{t}(\Omega)\right) \sim \lambda(\Omega)-t \int_{\partial \Omega}\left|\partial_{\nu} u\right|^{2} v \cdot \nu d \Gamma .
$$

Here the domain $\Omega$ is perturbed by the family of smooth mappings $h_{t}: \mathbb{R}^{n} \mapsto \mathbb{R}^{n}$ such that $h_{0}$ is the identical mapping; $v=d h_{t} /\left.d t\right|_{t=0}, \nu$ is the outward unit normal on $\partial \Omega ; d \Gamma$ is the standard measure on the boundary; $\lambda(\Omega)$ denotes the first eigenvalue of the Dirichlet Laplacian in a bounded domain $\Omega \subset \mathbb{R}^{n}$, and $u$ is the eigenfunction corresponding to $\lambda(\Omega)$ and normalized in the Lebesgue space 
$L^{2}(\Omega)$. Subsequently, this formula was generalized in order to make it applicable to an arbitrary eigenvalue of a second-order system, but the requirement that perturbations of the reference domain are described by a family of smooth isomorphisms remained unchanged (see [14], [4], [7], [2] and references therein). On the other hand, there are many problems in which more general classes of perturbations are involved, namely, non-smooth perturbations and perturbations that cannot be described by a class of isomorphisms. An example of such a problem is the shape optimization problem with perturbations subject to certain constraints.

In [10] and [11], a new abstract approach was proposed that allows to treat such problems. In particular, an example of the perturbation of the first eigenvalue of the Dirichlet Laplacian was used in [10] to demonstrate that the abstract Theorem 1 proved in this paper is applicable to non-smooth reference domains as well as to non-smooth perturbations.

In the present paper, we discuss perturbations of an arbitrary eigenvalue of the second-order matrix elliptic operator which is formally self-adjoint and is acting on vector functions subject to the homogeneous Dirichlet condition on the boundary of a domain satisfying minimal smoothness and geometrical assumptions. Minimal assumptions are imposed on the perturbations as well; in particular, they are not supposed to be given by a family of isomorphisms.

Let $\Omega_{1}$ and $\Omega_{2}$ be bounded domains with nonempty intersection and with boundaries $\Gamma_{1}$ and $\Gamma_{2}$, respectively. We introduce the following sesquilinear forms:

$$
(u, v)_{k} \equiv(u, v)_{\Omega_{k}}=\sum_{i, j=1}^{n} \int_{\Omega_{k}}\left(A_{i j} \partial_{x_{j}} u, \partial_{x_{i}} v\right) d x, \quad k=1,2 .
$$

Here $(\cdot, \cdot)$ is the standard inner product in $\mathbb{C}^{M}$, the constant $M \times M$-matrices $A_{i j}$ are such that $A_{i j}=A_{j i}^{*}$, where $A_{j i}^{*}$ stands for the adjoint matrix. By

$$
\mathcal{A}\left(\partial_{x}\right)=-\sum_{i, j=1}^{n} A_{i j} \partial_{x_{i}} \partial_{x_{j}}
$$

we denote the corresponding differential operator; the matrix $-\mathcal{A}(\xi)$ is supposed to be positive definite for all $\xi \in \mathbb{R}^{n} \backslash \mathcal{O}$.

Let us consider the following spectral problems formulated in the variational form:

$$
(\varphi, v)_{1}=\lambda\langle\varphi, v\rangle_{1} \quad \text { for all } v \in\left(\stackrel{\circ}{W}^{1,2}\left(\Omega_{1}\right)\right)^{M}
$$

and

$$
(U, V)_{2}=\mu\langle U, V\rangle_{2} \text { for all } V \in\left(\stackrel{\circ}{W}^{1,2}\left(\Omega_{2}\right)\right)^{M} .
$$

Here $\langle\cdot, \cdot\rangle_{k}$ is the inner product in $\left(L^{2}\left(\Omega_{k}\right)\right)^{M}, \varphi \in\left(\stackrel{\circ}{W}^{1,2}\left(\Omega_{1}\right)\right)^{M}$ and $U \in$ $\left(\stackrel{\circ}{W}^{1,2}\left(\Omega_{2}\right)\right)^{M}$. The space $\mathscr{W}^{1,2}\left(\Omega_{j}\right)$ is, as usual, the closure of $C_{0}^{\infty}\left(\Omega_{j}\right)$ in the norm of $W^{1,2}\left(\Omega_{j}\right)$. 
Assumption I. We suppose that $\Omega_{1}$ is a bounded Lipschitz domain and $\Omega_{2}$ is an arbitrary domain such that $\Omega_{2} \backslash \Omega_{1}$ and $\Omega_{1} \backslash \Omega_{2}$ belong to the $d$ neighborhood of $\Gamma_{1}$ with a small $d>0$.

Note that the perturbed domain $\Omega_{2}$ can be arbitrary (and not assumed to be Lipschitz).

Let $\lambda_{m}$ be the $m$ th eigenvalue of problem (2), by $X_{m}$ we denote the corresponding eigenspace, $\operatorname{dim} X_{m}=J_{m}$. According to [10], if $d$ is sufficiently small, then there exist exactly $J_{m}$ eigenvalues of problem (3) within the interval $\left(\left(\lambda_{m-1}+\lambda_{m}\right) / 2,\left(\lambda_{m}+\lambda_{m+1}\right) / 2\right)$; these eigenvalues are denoted by $\mu_{1}, \ldots, \mu_{J_{m}}$. One of our main results is the following

Theorem 1 If Assumption I holds, then the following asymptotic formula is valid:

$$
\frac{1}{\mu_{j}}=\frac{1}{\lambda_{m}}+\tau_{j}+O\left(d^{1+\delta}\right), \quad j=1, \ldots, J_{m} .
$$

Here $\delta$ is a positive number depending only on $\Omega_{1}$ and the matrices $A_{i j} ;\left\{\tau_{j}\right\}_{j=1}^{J_{m}}$ is the set of eigenvalues of the following finite-dimensional eigenvalue problem:

$$
\frac{1}{\lambda_{m}}\left(\left(\Psi_{\varphi}, \Psi_{\psi}\right)_{2}-\left(\Phi_{\varphi}, \Phi_{\psi}\right)_{2}-(\varphi, \psi)_{\Omega_{1} \backslash \Omega_{2}}-\left(\Psi_{\varphi}, \psi\right)_{2}-\left(\Psi_{\psi}, \varphi\right)_{2}\right)=\tau(\varphi, \psi)_{1}
$$

for all $\psi \in X_{m}$, where the function $\Phi=\Phi_{\varphi}$ depends on $\varphi \in X_{m}$ and is such that the equality

$$
(\Phi, w)_{2}=0 \quad \text { holds for all } w \in\left(\stackrel{\circ}{W}^{1,2}\left(\Omega_{2}\right)\right)^{M}
$$

and $\Phi+\varphi \in\left(\stackrel{\circ}{W}^{1,2}\left(\Omega_{2}\right)\right)^{M}$. Furthermore, the function $\Psi=\Psi_{\varphi} \in\left(\stackrel{\circ}{W}^{1,2}\left(\Omega_{2}\right)\right)^{M}$ is a solution of

$$
(\Psi, w)_{2}=(\varphi, w)_{2}-\lambda_{m}\langle\varphi, w\rangle_{2}=\int_{\Gamma_{1}}(N \varphi, w) d \Gamma \quad \forall w \in\left(\stackrel{\circ}{W^{1,2}}\left(\Omega_{2}\right)\right)^{M},
$$

where

$$
N \varphi=\sum_{i, j=1}^{n} A_{i j} \nu_{j} \partial_{x_{i}} \varphi
$$

is the co-normal derivative and $\nu$ is the outward unit normal to $\Gamma_{1}$.

Similar assertion was proved in [10] for the Laplace operator under the additional assumption that the boundary of perturbed domain $\Omega_{2}$ is also Lipschitz. Thus, Theorem 1 covers a larger set of perturbed domains even for the Laplacian.

The next theorem deals with the remainder in the asymptotic formula (4).

Theorem 2 Suppose that the boundary $\Gamma_{1}$ is of class $C^{1}$. If the following positivity assumption

$$
\sum_{i, j=1}^{n}\left(A_{i j} \xi_{i}, \xi_{j}\right) \geq 0 \text { for all } \xi_{i} \in \mathbb{C}^{M}, i=1, \ldots, n
$$

holds for the operator $\mathcal{A}$, then formula (4) is valid for an arbitrary $\delta \in(0,1)$. 
In the next theorem we give conditions on the reference and perturbed domains that guarantee the validity of the Hadamard formula for perturbed eigenvalues. Below we use the following coordinates near the boundary $\Gamma_{1}: \tau$ is the signed distance from $x$ to $\Gamma_{1}$ (it is negative for $x \in \Omega_{1}$ ) and $z \in \Gamma_{1}$.

Theorem 3 Let $\Omega_{1}$ be of class $C^{1, \alpha}, \alpha \in(0,1)$, and let $\tau=h(z, d)$ be the equation of $\Gamma_{2}$, where $h$ satisfies

$$
\int_{\Gamma_{1}}\left|\nabla_{z} h(z, d)\right|^{2} d \Gamma=o(1) \quad \text { as } d \rightarrow 0 .
$$

Then the following formula holds:

$$
\frac{1}{\mu_{j}}=\frac{1}{\lambda_{m}}+\theta_{j}+o(d),
$$

where $\left\{\theta_{j}\right\}_{j=1}^{J_{m}}$ are eigenvalues of the problem

$$
\frac{1}{\lambda_{m}} \int_{\Gamma_{1}} h(z, d) \sum_{i, j=1}^{n}\left(A_{i j} \nu_{j} \nu_{i} \partial_{\nu} \varphi, \partial_{\nu} \psi\right) d \Gamma=\theta(\varphi, \psi)_{1} \quad \text { for all } \psi \in X_{m} .
$$

The last formula extends the classical Hadamard formula to second-order matrix operators and is new even for the Laplacian as well.

If $\Omega_{2} \subset \Omega_{1}$, then $\Psi=0$ in Theorem 1 . Let the boundary of $\Omega_{2}$ be also Lipschitz. By [5, Theorem 4.2],

$$
\int_{\Omega_{2}}\left|\nabla \Phi_{\varphi}\right| d x \leq C \int_{\Gamma_{2}}\left|\nabla_{\tau} \varphi\right|^{2} d \Gamma
$$

where $\nabla_{\tau}$ is the tangent gradient on $\Gamma_{2}$. The constant $C$ depends on the number of fixed-size cylinders covering $\Gamma_{2}$ and on the local Lipschitz constant of functions that give local representations of the domain as an upper graph of Lipschitz functions. Using (12), one can write the asymptotic formula (4) as follows:

$$
\frac{1}{\mu_{j}}=\frac{1}{\lambda_{m}}-\frac{\kappa_{j}}{\lambda_{m}^{2}}+O\left(d^{1+\delta}\right)+O(q) .
$$

Here

$$
q=\max \int_{\Gamma_{2}}\left|\nabla_{\tau} \varphi\right|^{2} d \Gamma,
$$

the maximum taken over all $\varphi \in X_{m}$ such that $(\varphi, \varphi)_{1}=1 ; \kappa_{j}, j=1, \ldots, J_{m}$, are eigenvalues of the problem

$$
\sum_{i, j=1}^{n} \int_{\Omega_{1} \backslash \Omega_{2}}\left(A_{i j} \partial_{x_{j}} \varphi, \partial_{x_{i}} \psi\right) d x=\kappa \int_{\Omega_{1}}(\varphi, \psi) d x \text { for all } \psi \in X_{m},
$$

where $\varphi \in X_{m}$. If $\Gamma_{1}$ is smooth and $q=o(d)$, then these formulae imply (10). In Sect. 3.6, we consider a two-dimensional domain whose boundary has 

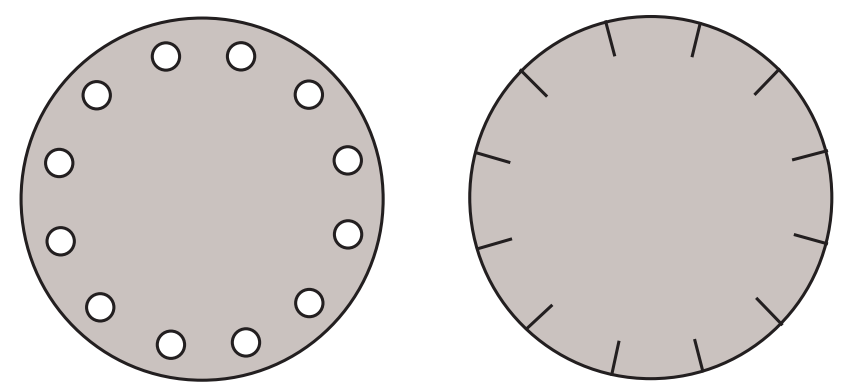

Figure 1: Perturbations by small circular and curvilinear cuts

corner points. In this case one cannot represent the boundary of perturbed domain through the normal shift and therefore the Hadamard formula cannot be applied. However, formula

$$
\frac{1}{\mu_{j}}=\frac{1}{\lambda_{m}}-\frac{\kappa_{j}}{\lambda_{m}^{2}}+O(d)
$$

still holds in this case; here $\kappa_{j}, j=1, \ldots, J_{m}$, are eigenvalues of (14).

In the last section, we apply the general formula to the case when a smooth bounded domain is perturbed either by curvilinear cuts, whose lengths are of the same order as the distances between them, or by circular cuts uniformly distributed along the boundary, see Fig.1. Of course, the Hadamard formula is not valid in these cases, because there are no smooth mappings from the unperturbed domains onto perturbed ones. Let us describe the first of these examples for the Laplace operator. Let $\Omega_{1}$ be a $2 \mathrm{D}$ simply connected domain whose boundary has length 1 . The perturbed domain is defined by

$$
\Omega_{2}=\Omega \backslash \cup_{j=1}^{N} \Upsilon_{j}^{\varepsilon}, \quad \Upsilon_{j}^{\varepsilon}=\{x \in \Omega \cap \mathcal{V}: z=j \varepsilon, \tau \in[-\varepsilon \ell(z), 0]\},
$$

where $z$ is the arc length measured from a certain fixed point and $\ell(z)$ is a positive $C^{\infty}$ function on $\Gamma_{1}$. In this case, the function $\Psi$ vanishes in (5) and the function $\Phi$ contributes to the asymptotics of $\mu$. Assuming that $\mu$ is a simple eigenvalue located near the eigenvalue $\lambda$ and the corresponding eigenfunction $\varphi$ is normalized so that $\|\varphi\|_{L^{2}\left(\Omega_{1}\right)}=1$, we get

$$
\frac{1}{\mu}=\frac{1}{\lambda}-\varepsilon \frac{\tau}{\lambda^{2}}+O\left(\varepsilon^{1+\delta}\right) .
$$

Here $\delta$ is an arbitrary number from the interval $(0,1)$ and

$$
\tau=\int_{\Gamma_{1}} \int_{-1 / 2}^{1 / 2} \int_{-\infty}^{0}\left|\nabla_{\xi} w(\xi, z)\right|^{2} d \xi d z
$$

where $w$ is a bounded solution of the following problem:

$$
\Delta_{\xi} w=0 \text { in } \Pi_{z}=\left\{\xi=\left(\xi_{1}, \xi_{2}\right): \xi<0, \xi_{2} \in(-1 / 2,1 / 2)\right\} \backslash \mathcal{L}_{z},
$$


$\mathcal{L}_{z}=\left\{\xi=\left(\xi_{1}, 0\right): \xi_{1} \in(-\ell(z), 0)\right\}$. Moreover, $w$ must satisfy the boundary conditions:

$$
\begin{gathered}
w\left(0, \xi_{2}, z\right)=0, \quad \xi_{2} \in(-1 / 2,1 / 2), \\
w\left(\xi_{1}, 0, z\right)=-\xi_{1} \partial_{\nu} \varphi(0, z), \quad \xi_{1}>-\ell(z),
\end{gathered}
$$

and the following periodicity conditions

$$
w\left(\xi_{1},-1 / 2, z\right)=w\left(\xi_{1}, 1 / 2, z\right), \quad \partial_{\xi_{2}} w\left(\xi_{1},-1 / 2, z\right)=\partial_{\xi_{2}} w\left(\xi_{1}, 1 / 2, z\right), \quad \xi_{1}<0 .
$$

Other examples showing importance of generalized Hadamard formula are presented in Sect.4, where we consider both types of perturbations shown in Fig. 1 in the case of second order systems.

Proofs of above asymptotic formulae are based on the abstract asymptotic formula obtained in [10] for eigenvalues of compact operators acting in various Hilbert spaces. However, further analysis differs essentially from that in [10]. Indeed, here we apply Hardy type inequalities instead of $L^{p}$-estimates valid for solutions of the Dirichlet problem in Lipschitz domains. The distance from the boundary of $2 d$-neighborhood of $\Omega_{1}$ is taken as a weight function in the Hardy inequality. It does not depend on the perturbed domain which allows us to show in Lemma 3 that for domains close to $\Omega_{1}$ there is always an interval for powers of the weight in which the Dirichlet problem in $\Omega_{2}$ is solvable. Thus, we avoid imposing any smoothness assumption on $\Omega_{2}$. Another important tool is based on the fact that a weak solution in a Lipschitz domain has some additional smoothness. One more advantage of the method is that it does not require the use of an extension operator, see [10, Sect. 4.3]. This allows us to obtain all results under rather weak assumptions about smoothness of $\Omega_{1}$ and $\Omega_{2}$, which are new even for the Dirichlet Laplacian.

\section{General domains}

In this section, we consider eigenvalue problems (2) and (3) assuming that $\Omega_{1}$ and $\Omega_{2}$ are bounded domains with non-empty intersection. The proximity of domains and operators is described by smallness of constants in some integral inequalities. The main result is obtained in Theorem 5, where an asymptotic representation is given for eigenvalues of problem (3) located near an eigenvalue of problem (2).

For convenience of the readers, we present in Sect. 2.1 an asymptotic result from [10] about eigenvalues of two compact operators acting in different Hilbert spaces. In Sect. 2.2, we apply this abstract theorem to problems (2) and (3).

\subsection{Abstract theorem on asymptotics}

Let $K_{j}, j=1,2$, be a compact, non-negative, self-adjoint operator in a Hilbert space $H_{j}$ with the inner product $(\cdot, \cdot)_{j}$ and with the norm $\|\cdot\|_{j}$. By $\lambda_{k}^{-1}, k=$ $1,2, \ldots$, we denote the eigenvalues of $K_{1}$ numbered so that $0<\lambda_{1}<\lambda_{2}<\cdots$; the eigenspace corresponding to $\lambda_{k}$ is denoted by $X_{k}$, and $J_{k}=\operatorname{dim} X_{k}$. The 
notation $\lambda_{k}^{-1}$ used for eigenvalues of a compact operator is applied because we reserve the notation $\lambda_{k}$ for eigenvalues of the corresponding differential operator.

Our aim is to describe the set of those eigenvalues of the operator $K_{2}$ that are located near an eigenvalue $\lambda_{m}^{-1}$ of the operator $K_{1}$. In order to measure the proximity of these eigenvalues we introduce a bounded, linear operator $S$ : $H_{1} \rightarrow H_{2}$, a positive constant $c_{1}$ and an integer $N$ such that $N \geq m$ and

$$
\frac{c_{1}\|S\|_{H_{1} \rightarrow H_{2}}^{2}}{\lambda_{N+1}} \leq \frac{1}{\lambda_{m+1}} .
$$

We set

$$
\mathcal{X}_{N}=\bigoplus_{j=1}^{N} X_{j}
$$

and denote by $\mathcal{Y}_{N}$ the orthogonal complement in $H_{2}$ to $S \mathcal{X}_{N}$. The proximity of the operators $K_{1}$ and $K_{2}$ is measured by the constant $\varepsilon$ in the following three inequalities:

$$
\left(K_{2} w, w\right)_{2} \leq c_{1}\left(K_{1} S^{*} w, S^{*} w\right)_{1}+\varepsilon\|w\|_{2}^{2} \text { for all } w \in \mathcal{Y}_{N},
$$

where $S^{*}: H_{2} \rightarrow H_{1}$ is the operator adjoint to $S$;

$$
||\left|S \varphi\left\|_{2}^{2}-\right\| \varphi\left\|_{1}^{2} \mid \leq \varepsilon\right\| \varphi \|_{1}^{2} \text { for all } \varphi \in \mathcal{X}_{N}\right.
$$

and

$$
\left|(B \varphi, w)_{2}\right| \leq \varepsilon\|\varphi\|_{1}\|w\|_{2} \text { for all } \varphi \in \mathcal{X}_{N} \text { and } w \in H_{2}
$$

where

$$
B=K_{2} S-S K_{1}: H_{1} \rightarrow H_{2} .
$$

Of course, all these inequalities hold provided $\varepsilon$ is sufficiently large, but it occurs that if $\varepsilon$ is small, then there are exactly $J_{m}$ eigenvalues of the operator $K_{2}$ in a neighborhood of $\lambda_{m}^{-1}$ (say in $\left(\frac{2}{\lambda_{m}+\lambda_{m+1}}, \frac{2}{\lambda_{m}+\lambda_{m-1}}\right)$ ). Moreover, these eigenvalues belong to $\left(\lambda_{m}^{-1}-c \varepsilon, \lambda_{m}^{-1}+c \varepsilon\right)$, where $c$ is a constant depending only on $\lambda_{m-1}$, $\lambda_{m}$ and $\lambda_{m+1}$. We denote these eigenvalues of $K_{2}$ by $\mu_{1}^{-1}, \ldots, \mu_{J_{m}}^{-1}$.

In order to describe the eigenvalues of the operator $K_{2}$ that are close to $\lambda_{m}^{-1}$ more explicitly, we introduce the constants $\rho_{m}$ and $\sigma_{m}$ that appear in the inequalities

$$
\left|(B \varphi, S \psi)_{2}\right| \leq \rho_{m}|| \varphi\left\|_{1}\right\| S \psi \|_{2} \text { for all } \varphi, \psi \in X_{m}
$$

and

$$
\left|(B \varphi, w)_{2}\right| \leq \sigma_{m}|| \varphi\left\|_{1}\right\| w \|_{2} \text { for all } \varphi \in X_{m}, w \in Y_{m},
$$

where $Y_{m}$ is the orthogonal complement to $S X_{m}$ in $H_{2}$. It is clear that we can put $\rho_{m}=\sigma_{m}=\varepsilon$, but, in fact, these constants can be essentially smaller than $\varepsilon$. In what follows, we denote by $P_{m}$ the orthogonal projector in $H_{2}$ onto $S X_{m}$, and so $Q_{m}=I-P_{m}$ is the orthogonal projector onto $Y_{m}$. 
Theorem 4 ([10]) There exists a constant $\varepsilon_{m}$ depending only on $\lambda_{m}$ and $\lambda_{m \pm 1}$ such that if (19) (20) and (21) hold for $\varepsilon \leq \varepsilon_{0}$, then for the eigenvalues $\mu_{s}^{-1}$, $s=1, \ldots, J_{m}$, of the operator $K_{2}$ introduced above the following asymptotic representation holds:

$$
\frac{1}{\mu_{j}}=\frac{1}{\lambda_{m}}+\omega_{j}+O\left(\sigma_{m}^{2}\left(\rho_{m}+\sigma_{m}^{2}\right)\right),
$$

where $\left\{\omega_{j}\right\}_{j=1}^{J_{m}}$ are eigenvalues of the problem

$$
(B \varphi, S \psi)_{2}+(\mathcal{R}(\varphi), B \psi)_{2}=\omega(S \varphi, S \psi)_{2} \quad \text { for all } \psi \in X_{m} .
$$

Here $\varphi \in X_{m}, \rho_{m}$ and $\sigma_{m}$ are the constants in (23) and (24), respectively, and $\mathcal{R}=\mathcal{R}(\varphi) \in Y_{m}$ is a solution of the equation

$$
\lambda_{m}^{-1} \mathcal{R}-Q_{m} K_{2} \mathcal{R}=Q_{m} B \varphi .
$$

This solution satisfies the estimate

$$
\|\mathcal{R}\|_{2} \leq C \sigma_{m}\|\varphi\|_{1} .
$$

(The factor $\|\varphi\|_{1}$ is lost in the corresponding formula (19) in [10, Theorem 1].)

\subsection{Eigenvalues of problem (3) located near $\lambda_{m}$}

In what follows, vector functions from $\stackrel{\circ}{W}^{1,2}\left(\Omega_{k}\right)^{M}$ and $\stackrel{\circ}{W}^{1,2}\left(\Omega_{1} \cap \Omega_{2}\right)^{M}$ are extended by zero outside $\Omega_{k}$ and $\Omega_{1} \cap \Omega_{2}$, respectively.

Let us reduce the spectral problems (2) and (3) to equivalent spectral problems for compact operators. The operators $K_{1}$ and $K_{2}$ are defined by the equality

$$
\left(K_{s} u, v\right)_{s}=\langle u, v\rangle_{s} \text { that holds for all } u, v \in \stackrel{\circ}{W}^{1,2}\left(\Omega_{s}\right)^{M} .
$$

The operator $K_{s}$ is compact and problems (2) and (3) take the form

$$
K_{1} \varphi=\lambda^{-1} \varphi \quad \varphi \in \stackrel{\circ}{W}^{1,2}\left(\Omega_{1}\right)^{M},
$$

and

$$
K_{2} U=\mu^{-1} U \quad U \in \stackrel{\circ}{W}^{1,2}\left(\Omega_{2}\right)^{M},
$$

respectively. In order to compare eigenvalues of these problems we apply Theorem 4 . We put $H_{k}=\stackrel{\circ}{W}^{1,2}\left(\Omega_{k}\right)^{M}$ and supply this space with the inner product $(\cdot, \cdot)_{k}$, the corresponding norm is denoted by $\|\cdot\|_{k}$. We define the operator $S: H_{1} \rightarrow H_{2}$ as follows. Let $\varphi \in H_{1}$, then $u=S \varphi \in H_{2}$ is a unique solution of the problem

$$
(u, w)_{2}=(\varphi, w)_{2} \text { for all } w \in H_{2} .
$$

The adjoint operator $S^{*}: H_{2} \rightarrow H_{1}$ is defined by

$$
\left(\varphi, S^{*} w\right)_{1}=(\varphi, w)_{1}
$$


for all $\varphi \in H_{1}$. Clearly,

$$
\|S\|_{H_{1} \rightarrow H_{2}}=\left\|S^{*}\right\|_{H_{2} \rightarrow H_{1}}=1 .
$$

Moreover, if $w \in H_{1} \cap H_{2}$, then $S w=w$ and $S^{*} w=w$. If $w_{k} \in H_{k}$ and $w_{k}$ is orthogonal to $H_{1} \cap H_{2}$, then the orthogonality is also valid for $S w_{1}$ and $S^{*} w_{2}$.

Let $0<\lambda_{1}<\lambda_{2}<\cdots$ be eigenvalues of problem (2) or, equivalently, $\lambda_{j}^{-1}$ are eigenvalues of problem (29), and let $J_{j}$ be the multiplicity of $\lambda_{j}$. By $X_{j}$ we denote the space of eigenfunctions corresponding to the eigenvalue $\lambda_{j}$.

The results in this section are valid without Assumption I made in Introduction. It is sufficient to assume that $\Omega_{1}$ and $\Omega_{2}$ are two bounded domains with a non-empty intersection and the following inequality

$$
\int_{\Omega_{k}}|w|^{2} d x \leq \sigma\|w\|_{k}^{2}
$$

is valid with a small constant $\sigma$ for all $w \in H_{k}, k=1,2$, orthogonal to $H_{1} \cap H_{2}$. The latter constant $\sigma$ describes the proximity of $\Omega_{1}$ and $\Omega_{2}$, cf. [10].

We introduce $\rho_{1}$ as the best constant in the inequality

$$
\left|(B \varphi, S \psi)_{2}\right| \leq \rho_{1}\|\varphi\|_{1}\|S \psi\|_{2}
$$

that holds for $B$ given by (22) and for all $\varphi, \psi \in X_{m}$. Let $Y_{m}$ be the orthogonal complement of $S X_{m}$ in $H_{2}$, let also $Q_{m}=I-P_{m}$, where $P_{m}$ denotes the orthogonal projector on $S X_{m}$ in $H_{2}$. By $(u, v)_{\Omega}$ we denote the sesquilinear form (1) defined on $\Omega$, and $\langle u, v\rangle_{\Omega}=\int_{\Omega}(u, v) d x$. In particular, $(u, v)_{\Omega_{k}}=(u, v)_{k}$ and $\langle u, v\rangle_{\Omega_{k}}=\langle u, v\rangle_{k}$.

Theorem 5 Let $\Omega_{1}$ and $\Omega_{2}$ be two domains with non-empty intersection, and let the constant $\sigma$ in (33) be small. Then there are exactly $J_{m}$ eigenvalues of problem (3) in the interval $\left(\left(\lambda_{m-1}+\lambda_{m}\right) / 2,\left(\lambda_{m}+\lambda_{m+1}\right) / 2\right)$, for which the following asymptotic formula holds:

$$
\frac{1}{\mu_{j}}=\frac{1}{\lambda_{m}}+\omega_{j}+O\left(\sigma\left(\rho_{1}+\sigma\right)\right) .
$$

Here $\left\{\omega_{j}\right\}_{j=1}^{J_{m}}$ are the eigenvalues of problem (26), where $\varphi \in X_{m}$ and $\mathcal{R}=$ $\mathcal{R}(\varphi) \in Y_{m}$ is a solution of equation (27).

Proof. In order to apply Theorem 4 we have to verify conditions (19), (20) and (21).

Let us prove inequality (19) with $c_{1}=1$ and $\varepsilon=2 \sqrt{2 \sigma c_{0}}$, where $c_{0}$ is the constant in

$$
\int_{\Omega_{2}}|\varphi|^{2} d x \leq c_{0}(\varphi, \varphi)_{2}, \quad \varphi \in H_{2} .
$$

This inequality follows from

$$
\left(K_{2} w, w\right)_{2} \leq\left(K_{1} S^{*} w, S^{*} w\right)_{1}+\varepsilon\|w\|_{2}^{2} \quad \text { valid for all } w \in H_{2} .
$$


Using the definition of $K_{1}$ and $K_{2}$, we write the last inequality as follows:

$$
\int_{\Omega_{2}}|w(x)|^{2} d x \leq \int_{\Omega_{1}}\left|S^{*} w(x)\right|^{2} d x+\varepsilon(w, w)_{2} \quad \text { for all } w \in H_{2} .
$$

In order to prove (37), we split $w \in H_{2}$ into the sum

$$
w=w_{0}+w_{1}, \quad \text { where } w_{0} \in H_{1} \cap H_{2} \text { and } w_{1} \text { is orthogonal to } H_{1} \cap H_{2} \text {. }
$$

Then inequality (37) takes the form

$$
\int_{\Omega_{2}}\left|w_{0}+w_{1}\right|^{2} d x \leq \int_{\Omega_{1} \cap \Omega_{2}}\left|w_{0}+S^{*} w_{1}\right|^{2} d x+\varepsilon\left(w_{0}, w_{0}\right)_{2}+\varepsilon\left(w_{1}, w_{1}\right)_{2} .
$$

Using (33) for $S^{*} w_{1}$, we obtain

$$
\int_{\Omega_{1}}\left|S^{*} w_{1}\right|^{2} d x \leq \sigma\left(S^{*} w_{1}, S^{*} w_{1}\right)_{1} \leq \sigma\left(w_{1}, w_{1}\right)_{2} .
$$

Applying (33) to $w_{1}$, we get that

$$
\int_{\Omega_{2}}\left|w_{1}\right|^{2} d x \leq \sigma\left(w_{1}, w_{1}\right)_{2} .
$$

Since

$$
\begin{aligned}
& \int_{\Omega_{2}}\left|w_{0}+w_{1}\right|^{2} d x=\int_{\Omega_{2}}\left(\left|w_{0}\right|^{2}+\left|w_{1}\right|^{2}+2 \Re\left(w_{0}, w_{1}\right)\right) d x=\int_{\Omega_{1}}\left|w_{0}+S^{*} w_{1}\right|^{2} d x \\
& +\int_{\Omega_{2}}\left(2 \Re\left(w_{0}, w_{1}\right)-2 \Re\left(w_{0}, S^{*} w_{1}\right)+\left|w_{1}\right|^{2}-\left|S^{*} w_{1}(x)\right|^{2}\right) d x,
\end{aligned}
$$

we have that

$$
\int_{\Omega_{2}}\left|w_{0}+w_{1}\right|^{2} d x \leq \int_{\Omega_{2}}\left(\left|w_{0}+S^{*} w_{1}\right|^{2}+2 \delta\left|w_{0}\right|^{2}\right) d x+\frac{2 \sigma(1+\delta)}{\delta}\left(w_{1}, w_{1}\right)_{2} .
$$

Here (40) and (41) are also taken into account. Using (36) and choosing $\varepsilon=2 c_{0} \delta$ and $\delta=\sqrt{2 \sigma c_{0}^{-1}}$, we arrive at (39), and so (19) is valid.

In particular, $c_{1}=1$ in our case, and so we can take $N=m$ in (18).

Let us verify (20) for $\varphi \in \mathcal{X}_{m}=X_{1} \oplus \cdots \oplus X_{m}$. Clearly, we have that $\|S \varphi\|_{2} \leq\|\varphi\|_{1}$. Let us estimate $\|S \varphi\|_{2}$ from below. We represent $\varphi$ as the sum

$$
\varphi=\varphi_{0}+\varphi_{1}, \quad \text { where } \varphi_{0} \in H_{1} \cap H_{2} \text { and } \varphi_{1} \text { is orthogonal to } H_{1} \cap H_{2} .
$$

Since $S \varphi=\varphi_{0}+S \varphi_{1}$ and $\|S \varphi\|_{2}^{2}=\left\|\varphi_{0}\right\|_{1}^{2}+\left\|S \varphi_{1}\right\|_{2}^{2}$, we have $\|S \varphi\|_{2} \geq\left\|\varphi_{0}\right\|_{1}$. Furthermore, (33) implies that

$$
\int_{\Omega_{1}}\left|\varphi_{1}\right|^{2} d x \leq \sigma\left(\varphi_{1}, \varphi_{1}\right)_{1} .
$$


Let $\varphi=\Phi_{1}+\cdots+\Phi_{m}$, where $\Phi_{j} \in X_{j}$. From $\left(\varphi_{1}, \varphi_{1}\right)_{1}=\left(\varphi, \varphi_{1}\right)_{1}$ it follows that

$$
\left(\varphi_{1}, \varphi_{1}\right)_{1}=\sum_{j=1}^{m}\left(\Phi_{j}, \varphi_{1}\right)_{1}=\sum_{j=1}^{m} \lambda_{j}\left\langle\Phi_{j}, \varphi_{1}\right\rangle_{1} \leq\left(\sum_{j=1}^{m} \lambda_{j}^{2}\left\|\Phi_{j}\right\|_{L^{2}\left(\Omega_{1}\right)}^{2}\right)^{1 / 2}\left\|\varphi_{1}\right\|_{L^{2}\left(\Omega_{1}\right)} .
$$

Since $\Phi_{j}$ is an eigenvector corresponding to $\lambda_{j}$, we get from (43) that

$$
\left(\varphi_{1}, \varphi_{1}\right)_{1} \leq \lambda_{m}^{1 / 2} \sigma^{1 / 2} a_{1}(\varphi, \varphi)
$$

Therefore,

$$
\|S \varphi\|_{2}^{2} \geq\left\|\varphi_{0}\right\|_{1}^{2}=\|\varphi\|_{1}^{2}-\left\|\varphi_{1}\right\|_{1}^{2} \geq\left(1-\lambda_{m}^{1 / 2} \sigma^{1 / 2}\right)\|\varphi\|_{1}^{2},
$$

and so (20) folds with $\varepsilon=\lambda_{m}^{1 / 2} \sigma^{1 / 2}$.

Finally, let us show that (21) holds with $\varepsilon=c \sigma^{1 / 2}$, or, equivalently, we have that

$$
\|B \varphi\|_{2} \leq c \sigma^{1 / 2}\|\varphi\|_{1} \text { for all } \varphi \in \mathcal{X}_{m} .
$$

Since

$$
(B \varphi, w)_{2}=\left(K_{2} S \varphi, w\right)_{2}-\left(S K_{1} \varphi, w\right)_{2}=\langle S \varphi, w\rangle_{2}-\left\langle\varphi, S^{*} w\right\rangle_{1}
$$

for $\varphi \in \mathcal{X}_{m}$ and $w \in H_{2}$, the representations (38) and (42) yield that

$$
(B \varphi, w)_{2}=\left\langle\varphi_{0}, w_{1}\right\rangle_{2}-\left\langle\varphi_{1}, w_{0}\right\rangle_{1}+\left\langle S \varphi_{1}, w\right\rangle_{2}-\left\langle\varphi, S^{*} w_{1}\right\rangle_{1} .
$$

Now, applying (33) to the functions $w_{1}, S \varphi_{1}, \varphi_{1}$ and $S^{*} w_{1}$, we arrive at

$$
\left|(B \varphi, w)_{2}\right| \leq c \sigma^{1 / 2}\|\varphi\|_{1}\|w\|_{2} \text { for } \varphi \in X_{1} \text { and } w \in H_{2},
$$

where $c$ is a constant depending on the constant $c_{0}$ in (36). Thus, (45), and consequently (21) are satisfied. Using results obtained in Sect.2.1, we conclude that there are exactly $J_{m}$ eigenvalues of problem (30) in the interval $\left(2 /\left(\lambda_{m+1}+\right.\right.$ $\left.\left.\lambda_{m}\right), 2 /\left(\lambda_{m}+\lambda_{m-1}\right)\right)$ and they are located in $\left(\lambda_{m}^{-1}-c \varepsilon, \lambda_{m}^{-1}+c \varepsilon\right)$, where $\varepsilon=c \sigma^{1 / 2}$ and the constant $c$ depends on $\lambda_{m-1}, \lambda_{m}$ and $\lambda_{m+1}$. This gives the first assertion of lemma.

In order to apply Theorem 4 , we put $\sigma_{m}=c \sigma^{1 / 2}$ and take $\rho_{m}$ equal to the constant $\rho_{1}$ in (34). This choice of constants yields formula (25) in the form (35). The proof of Lemma is complete.

Remark 1 It follows from (48) that the constant $\sigma_{m}$ is estimated by $c \sigma^{1 / 2}$. Using this along with (48) and (28), we obtain

$$
\left|(\mathcal{R}(\varphi), B \psi)_{2}\right| \leq c \sigma\|\varphi\|_{1}\|\psi\|_{1} \text { for } \varphi, \psi \in X_{m} .
$$

In conclusion of this section, we obtain a useful representation of $(B \varphi, S \psi)_{2}$ involved in (26). Let $\varphi, \psi \in X_{m}$, then we use the representations

$$
S \varphi=\varphi+\Phi_{\varphi} \text { and } S \psi=\psi+\Phi_{\psi},
$$


where the vector function $\Phi=\Phi_{\varphi}$ satisfies the integral identity $(\Phi, w)_{2}=0$ for all $w \in H_{2}, \Phi+\varphi \in H_{2}$ and the same is true for $\Phi_{\psi}$. Therefore,

$$
(B \varphi, S \psi)_{2}=\left\langle\varphi+\Phi_{\varphi}, \psi+\Phi_{\psi}\right\rangle_{2}-\lambda_{m}^{-1}\left(\varphi+\Phi_{\varphi}, \psi+\Phi_{\psi}\right)_{2} .
$$

From the definition of $\Phi_{\varphi}$ and $\Phi_{\psi}$, it follows that

$$
\left(\Phi_{\varphi}, \psi+\Phi_{\psi}\right)_{2}=\left(\varphi+\Phi_{\varphi}, \Phi_{\psi}\right)_{2}=0
$$

which implies

$$
(B \varphi, S \psi)_{2}=\left\langle\varphi+\Phi_{\varphi}, \psi+\Phi_{\psi}\right\rangle_{2}+\lambda_{m}^{-1}\left(\left(\Phi_{\varphi}, \Phi_{\psi}\right)_{2}-(\varphi, \psi)_{2}\right) .
$$

Using the relation

$$
\left(\Psi_{\phi}, S \psi\right)_{2}=\left(\Psi_{\phi}, \psi\right)_{2}=\left(\varphi, \Phi_{\psi}+\psi\right)_{2}-\lambda_{m}\left\langle\varphi, \Phi_{\psi}+\psi\right\rangle_{2}
$$

and similar equality with exchanged $\varphi$ and $\psi$, we obtain

$$
\begin{aligned}
& \left\langle\varphi, \Phi_{\psi}\right\rangle_{2}=\frac{1}{\lambda_{m}}\left(\left(\varphi, \psi+\Phi_{\psi}\right)_{2}-\left(\Psi_{\varphi}, \psi\right)_{2}\right)-\langle\varphi, \psi\rangle_{2} \\
& =\frac{1}{\lambda_{m}}\left((\varphi, \psi)_{2}-\left(\Phi_{\varphi}, \Phi_{\psi}\right)_{2}-\left(\Psi_{\varphi}, \psi\right)_{2}\right)-\langle\varphi, \psi\rangle_{2}
\end{aligned}
$$

where we used (51). Applying this relation (and corresponding relation with exchanged $\varphi$ and $\psi$ ) to (52), we get

$$
(B \varphi, S \psi)_{2}=-\langle\varphi, \psi\rangle_{2}+\left\langle\Phi_{\varphi}, \Phi_{\psi}\right\rangle_{2}-\lambda_{m}^{-1}\left(\left(\Phi_{\varphi}, \Phi_{\psi}\right)_{2}-(\varphi, \psi)_{2}+\left(\Psi_{\varphi}, \psi\right)_{2}+\left(\Psi_{\psi}, \varphi\right)_{2}\right) .
$$

Now using that $(\varphi, \psi)_{1}=\lambda\langle\varphi, \psi\rangle_{1}$, we arrive at

$$
\begin{aligned}
& (B \varphi, S \psi)_{2}=\langle\varphi, \psi\rangle_{\Omega_{1} \backslash \Omega_{2}}+\left\langle\Phi_{\varphi}, \Phi_{\psi}\right\rangle_{2} \\
& -\lambda_{m}^{-1}\left(\left(\Phi_{\varphi}, \Phi_{\psi}\right)_{2}+(\varphi, \psi)_{\Omega_{1} \backslash \Omega_{2}}+\left(\Psi_{\varphi}, \psi\right)_{2}+\left(\Psi_{\psi}, \varphi\right)_{2}\right) .
\end{aligned}
$$

\section{Domains satisfying Assumption I}

In Sect. 3.1 and 3.2, we present estimates used for proving the main asymptotic formulae. In Sect. 3.3, we derive the asymptotic formula (4). In Sect. 3.4, we show that if the boundary of $\Omega_{1}$ is of class $C^{1}$ and $\mathcal{A}$ satisfies assumption (8), then $\delta$ in (4) is an arbitrary number belonging to the interval $(0,1)$. In Sect. 3.5 , we give sufficient conditions that guarantee that the classical Hadamard formula is valid, and in Sect. 3.6, we consider perturbations of a plane domain with angular points on the boundary. 


\subsection{Corollaries of Assumption I and an estimate for $\sigma$}

Let $D_{d}^{+}=\left\{x: \operatorname{dist}\left(x, \Omega_{1}\right)<d\right\}$ and $D_{d}^{-}=\left\{x \in \Omega_{1}: \operatorname{dist}\left(x, \Gamma_{1}\right)>d\right\}$, and so $\Omega_{2} \subset D_{d}^{+}$and $D_{d}^{-} \subset \Omega_{1} \cap \Omega_{2}$ according to our assumptions. Furthermore, we put $r=r(x)=\operatorname{dist}\left(x, \partial D_{2 d}^{+}\right)$. If $\beta<1$, then the following Hardy inequality

$$
\int_{D_{2 d}^{+}} r^{\beta-2}|v|^{2} d x \leq C_{\beta} \int_{D_{2 d}^{+}} r^{\beta}|\nabla v|^{2} d x
$$

is valid for functions belonging to $C_{0}^{\infty}\left(D_{2 d}^{+}\right)$. This estimate is a consequence of the one-dimensional Hardy inequality combined with a local change of variables flatting the boundary.

In what follows, we fix a small positive number $d_{0}$ so that the domains $D_{2 d}^{+}$ and $D_{2 d}^{-}$are Lipschitz provided $0 \leq d \leq d_{0}$; moreover, they can be defined locally by functions with uniformly bounded Lipschitz constants using the same local coordinates.

Lemma 1 Let $w \in\left(\stackrel{\circ}{W}^{1,2}\left(D_{d}^{+}\right)\right)^{M}$, then

$$
\int_{D_{d}^{+} \backslash D_{d}^{-}}|w|^{2} d x \leq c d^{2} \int_{D_{d}^{+} \backslash D_{d}^{-}}|\nabla w|^{2} d x
$$

and

$$
\int_{\partial D_{d}^{-}}|w|^{2} d x \leq C d \int_{D_{d}^{+} \backslash D_{d}^{-}}|\nabla w|^{2} d x
$$

Here $C$ is a positive constant that may depend on $\Omega_{1}, d_{0}$ and $A_{i j}$.

Proof. First we prove (55). The domain $D_{d}^{+} \backslash D_{d}^{-}$is locally described by $\xi_{d}^{-}\left(y^{\prime}\right)<y_{n}<\xi_{d}^{+}\left(y^{\prime}\right)$, where $y_{n}=\xi_{d}^{-}\left(y^{\prime}\right)$ and $y_{n}=\xi_{d}^{+}\left(y^{\prime}\right)$ are the local equations of the boundaries of $D_{d}^{-}$and $D_{d}^{+}$, respectively. Using the Newton--Leibniz formula with respect to $y_{n}$, one directly verifies that

$$
\int_{\left|y^{\prime}\right|<r} \int_{\xi_{d}^{-}\left(y^{\prime}\right)}^{\xi_{d}^{+}\left(y^{\prime}\right)}|w|^{2} d y_{n} d y^{\prime} \leq C d^{2} \int_{\left|y^{\prime}\right|<r} \int_{\xi_{d}^{-}\left(y^{\prime}\right)}^{\xi_{d}^{+}\left(y^{\prime}\right)}|\nabla w|^{2} d y_{n} d y^{\prime}
$$

and

$$
\int_{\left|y^{\prime}\right|<r, y_{n}=\xi_{d}^{-}\left(y^{\prime}\right)}|w|^{2} d y^{\prime} \leq C d \int_{\left|y^{\prime}\right|<r} \int_{\xi_{d}^{-}\left(y^{\prime}\right)}^{\xi_{d}^{+}\left(y^{\prime}\right)}|\nabla w|^{2} d y_{n} d y^{\prime} .
$$

These inequalities yield (55) and (56), respectively.

Corollary 1 (i) Let $w \in\left(\stackrel{\circ}{W}^{1,2}\left(D_{d}^{+}\right)\right)^{M}$, and let $w$ be orthogonal to $\left(\stackrel{\circ}{W}^{1,2}\left(D_{d}^{-}\right)\right)^{M}$ with respect to the inner product $(\cdot, \cdot)_{D_{d}^{+}}$. Then

$$
\int_{D_{d}^{+}}|w|^{2} d x \leq C d \int_{D_{d}^{+} \backslash D_{d}^{-}}|\nabla w|^{2} d x
$$

(ii) The number $\sigma$ in (33) can be taken as cd. 
Proof. Since the function $w$ satisfies $\mathcal{A} w=0$ in $D_{d}^{-}$, it follows from [5, Theorem 2.1] that

$$
\int_{D_{d}^{-}}|w|^{2} d x \leq C \int_{\partial D_{d}^{-}}|w|^{2} d \Gamma
$$

This together with (55) and (56) leads to (57).

Assertion (ii) follows from (i) .

Lemma 2 Let $\varphi \in X_{m}$ and $\|\varphi\|_{2}=1$, then

(i)

$$
\int_{\Omega_{1} \backslash D_{2 d}^{-}}|\varphi|^{2} d x \leq c d^{3}, \quad \int_{\Omega_{1} \backslash D_{2 d}^{-}}|\nabla \varphi|^{2} d x \leq c d \quad \text { and } \int_{\Gamma_{1}}|\nabla \varphi|^{2} d \Gamma \leq c .
$$

(ii) Let $\Phi$ be the same as in (50), then

$$
\int_{\Omega_{2}}|\Phi|^{2} d x \leq c d^{2} \quad \text { and } \quad \int_{\Omega_{2}}|\nabla \Phi|^{2} d x \leq c d .
$$

Proof. (i) Let us write $\varphi=\Phi_{1}+\Phi_{2}$, where $\Phi_{1} \in\left(W^{3,2}\left(\mathbb{R}^{n}\right)\right)^{M}$ solves the problem $-\mathcal{A} \Phi_{1}=\lambda \varphi$ in $\mathbb{R}^{n}$, whereas the function $\Phi_{2}$ solves the boundary value problem $\mathcal{A} \Phi_{2}=0$ in $\Omega_{1}$ and $\Phi_{2}=-\Phi_{1}$ on $\Gamma_{1}$.

Let $\gamma_{\tau}=\left\{x \in \Omega_{1}: \operatorname{dist}\left(x, \Gamma_{1}\right)=\tau\right\}$, then the trace theorem yields

$$
\int_{\gamma_{\tau}}\left(\left|\Phi_{1}\right|^{2}+\left|\nabla \Phi_{1}\right|^{2}\right) d \gamma \leq c .
$$

Using this estimate with $\tau=0$ and [5, Theorem 4.2] for the function $\Phi_{2}$, we obtain that

$$
\int_{\gamma_{\tau}}\left|\nabla \Phi_{2}\right|^{2} d \gamma \leq c .
$$

These inequalities with $\tau=0$ imply the third estimate (58). Integrating the above inequalities with respect to $\tau$, we arrive at the second inequality (58). The first inequality (58) follows from the second one combined with (55).

(ii) Let $\tau$ be the normal variable and let $\zeta=\zeta(\tau)$ be a smooth function vanishing for $\tau \leq 1$ and equal to 1 for $\tau \geq 2$. Putting $\zeta_{d}(\tau)=\zeta(\tau / d)$, we seek $\Phi$ in the form $\zeta_{d} \varphi+\Psi$. Then $\Psi$ must satisfy the boundary value problem $\mathcal{A} \Psi=-\mathcal{A}\left(\zeta_{d} \varphi\right)$ in $\Omega_{2}$ and $\Psi=0$ on $\Gamma_{2}$. Hence we have

$$
(\Psi, \Psi)_{2}=\int_{\Omega_{2}}\left(-\left(A_{i j} \partial_{j} \zeta_{d} \varphi, \partial_{i} \Psi\right)+\left(A_{i j} \partial_{j} \zeta_{d} \partial_{i} \varphi, \Psi\right)-\lambda_{m}\left(\zeta_{d} \varphi, \Psi\right)\right) d x,
$$

and so

$$
\begin{aligned}
& (\Psi, \Psi)_{2} \leq c\left(d^{-1}|| \varphi \|_{L^{2}\left(\Omega_{1} \backslash D_{2 d}^{-}\right.}\right)|| \nabla \Psi \|_{L^{2}\left(\Omega_{2} \backslash D_{2 d}^{-}\right)} \\
& \left.+d^{-1}|| \nabla \varphi\left\|_{L^{2}\left(\Omega_{1} \backslash D_{2 d}^{-}\right)}|| \Psi\right\|_{L^{2}\left(\Omega_{2} \backslash D_{2 d}^{-}\right)}+\|\varphi\|_{L^{2}\left(\Omega_{1} \backslash D_{2 d}^{-}\right)}|| \Psi \|_{L^{2}\left(\Omega_{2} \backslash D_{2 d}^{-}\right)}\right) .
\end{aligned}
$$


Since estimate (55) gives

$$
(\Psi, \Psi)_{2} \leq c\|\nabla \varphi\|_{L^{2}\left(\Omega_{1} \backslash D_{2 d}^{-}\right)}^{2},
$$

the second inequality (59) follows from (58). Furthermore, in view of (55), (56) and the second inequality (59) we obtain

$$
\int_{\Omega_{2} \backslash D_{2 d}^{-}}|\Psi|^{2} d x \leq c d^{3}
$$

and

$$
\int_{\partial D_{2 d}^{-}}|\Psi|^{2} d x \leq c d^{2}
$$

Applying [5, Theorem 2.1] to the function $\Psi$ in $D_{2 d}^{-}$and using (61), we arrive at

$$
\int_{D_{2 d}^{-}}|\Psi|^{2} d x \leq c d^{2}
$$

which together with (60) leads to the first inequality (59).

The first estimates (58) and (59) combined with (53) immediately give

Corollary 2 The following relation holds:

$$
(B \varphi, S \psi)_{2}=\frac{-1}{\lambda_{m}}\left(\left(\Phi_{\varphi}, \Phi_{\psi}\right)_{2}+(\varphi, \psi)_{\Omega_{1} \backslash \Omega_{2}}+\left(\Psi_{\varphi}, \psi\right)_{2}+\left(\Psi_{\psi}, \varphi\right)_{2}\right)+O\left(d^{2}\right) .
$$

\subsection{Weighted estimates}

In a domain $\Omega$ subject to $D_{d}^{-} \subset \Omega \subset D_{d}^{+}$, we consider the problem

$$
(u, w)_{\Omega}=\sum_{i=1}^{n}\left\langle f_{k}, \partial_{k} w\right\rangle_{\Omega}+\left\langle f_{0}, w\right\rangle_{\Omega} \text { for all } w \in\left(\stackrel{\circ}{W}^{1,2}(\Omega)\right)^{M},
$$

where $f_{0}, f_{1}, \ldots, f_{n} \in\left(L^{2}(\Omega)\right)^{M}$ and $u \in\left(\stackrel{\circ}{W}^{1,2}(\Omega)\right)^{M}$.

Lemma 3 There exist a constant $\beta_{0} \in(0,1)$ such that if $u \in\left(\stackrel{\circ}{W}^{1,2}(\Omega)\right)^{M}$ is a solution of (63), then the following inequality

$$
\int_{\Omega} r^{\beta-2}|u|^{2} d x+\int_{\Omega} r^{\beta}|\nabla u|^{2} d x \leq C \int_{\Omega}\left(r^{\beta} \sum_{k=1}^{n}\left|f_{k}\right|^{2}+r^{\beta+2}\left|f_{0}\right|^{2}\right) d x,
$$

holds for $|\beta| \leq \beta_{0}$; here $C$ is a positive constant depending only on the constant $\beta_{0}$, the domain $\Omega$ and the coefficients $A_{i j}$. 
Proof. Taking $w=r^{\beta} u$ in (63) and setting $v=r^{\beta / 2} u$, we obtain

$$
\left(r^{-\beta / 2} v, r^{\beta / 2} v\right)_{\Omega}=\sum_{k=1}^{n}\left\langle f_{k}, \partial_{k} r^{\beta / 2} v\right\rangle_{\Omega}+\left\langle f_{0}, r^{\beta / 2} v\right\rangle_{\Omega} .
$$

Therefore, we have

$$
\begin{aligned}
& (v, v)_{\Omega}-\frac{\beta^{2}}{4} \int_{\Omega} r^{-2} \sum_{i, j=1}^{n}\left(A_{i j} \partial_{i} r \partial_{j} r v, v\right) d x=\sum_{i=1}^{n}\left\langle r^{\beta / 2} f_{k}, \partial_{k} v\right\rangle_{\Omega} \\
& +\frac{\beta}{2} \sum_{i=1}^{n}\left\langle r^{\beta / 2} f_{k}, v r^{-1} \partial_{k} r\right\rangle_{\Omega}+\left\langle r^{\beta / 2+1} f_{0}, r^{-1} v\right\rangle_{\Omega} .
\end{aligned}
$$

Using Hölder inequality together with (54), we arrive at

$$
\int_{\Omega}|\nabla v|^{2} d x \leq c_{1} C \int_{\Omega}\left(r^{\beta}|\nabla F|^{2}+r^{\beta+2}|f|^{2}\right) d x,
$$

provided $|\beta|$ is small. This together with (54) gives (64).

Applying Lemma 3 to the operators $S, S^{*}$ and to the projection operator onto $H_{1} \cap H_{2}$, one obtains the following

Corollary 3 There exists a constant $\beta_{0} \in(0,1)$ such that the following estimates are valid for $|\beta| \leq \beta_{0}$.

(i) Let $v \in H_{2}$, then

$$
\left\|S^{*} v\right\|_{\Omega_{1}, \beta} \leq C|| v \|_{\Omega_{2}, \beta} .
$$

(ii) Let $v \in H_{1}$, then

$$
\|S v\|_{\Omega_{2}, \beta} \leq C\|v\|_{\Omega_{1}, \beta} .
$$

(iii) Let $P$ be the orthogonal projector ( with respect to the inner product $(\cdot, \cdot)_{\Omega_{1} \cup \Omega_{2}}$ ) from $\stackrel{\circ}{W}^{1,2}\left(\Omega_{1} \cup \Omega_{2}\right)$ onto $\stackrel{\circ}{W}^{1,2}\left(\Omega_{1} \cap \Omega_{2}\right)$. If $v \in W^{1,2}\left(\Omega_{1} \cup \Omega_{2}\right)$, then

$$
\|P v\|_{\Omega_{1} \cap \Omega_{2}, \beta} \leq C|| v \|_{\Omega_{1} \cup \Omega_{2}, \beta} .
$$

Proof. The operators appearing in (i)-(iii) can be written in the form (63) with $f=0$, see (31) and (32) for the operators $S$ and $S^{*}$, respectively, and the operator $u=P v$ is defined by

$$
(u, w)_{\Omega_{1} \cap \Omega_{2}}=(v, w)_{\Omega_{1} \cap \Omega_{2}} .
$$

Therefore, assertions (i)-(iii) follow from Lemma 3. 


\subsection{Proof of Theorem 1}

Let us consider the term $(\mathcal{R}(\varphi), B \psi)_{2}$ in (27). Let us write $\mathcal{R}=\lambda_{m} Q_{m} B \varphi+\mathcal{R}_{1}$, where $\mathcal{R}_{1}=\mathcal{R}_{1}(\varphi)$ satisfies

$$
\lambda_{m}^{-1} \mathcal{R}_{1}-Q_{m} K_{2} \mathcal{R}_{1}=g, \quad g=\lambda_{m} Q_{m} K_{2} Q_{m} B \varphi .
$$

According to Remark 1, we have

$$
\left\|\mathcal{R}_{1}\right\|_{2} \leq C\|g\|_{2}
$$

Since the operators $Q_{m}$ and $K_{2}$ are bounded in $H_{2}$, we combine this fact and (45), thus obtaining

$$
\left\|\mathcal{R}_{1}\right\|_{2} \leq C d^{1 / 2}\|\varphi\|_{1}
$$

Next we rewrite equation (66) in the form

$$
\left(\mathcal{R}_{1}, w\right)_{2}=\lambda_{m}\left(K_{2} \mathcal{R}_{1}, Q_{m} w\right)_{2}+\lambda_{m}(g, w)_{2} \text { for all } w \in H_{2} .
$$

It occurs that the right-hand side of this equation is as follows:

$$
\lambda_{m}\left\langle\mathcal{R}_{1}, Q_{m} w\right\rangle_{2}+\lambda_{m}^{2}\left\langle Q_{m} B \varphi, Q_{m} w\right\rangle_{2}-\lambda_{m}\left\langle\left(\mathcal{R}_{1}+\lambda_{m} Q_{m} B \varphi\right), P_{m} w\right\rangle_{2} .
$$

In order to represent it in the form $\langle f, w\rangle_{2}$ with some $f$, we fix an orthonormal basis $\left\{\Phi_{k}\right\}_{k=1}^{J_{m}}$ in $S X_{m}$ and write

$$
P_{m} \psi=\sum_{k=1}^{J_{m}}\left(\psi, \Phi_{k}\right)_{2} \Phi_{k}
$$

Then we have

$$
\left(P_{m} v, w\right)_{2}=\sum_{k=1}^{J_{m}}\left(v, \Phi_{k}\right)_{2}\left(\Phi_{k}, w\right)_{2}=\lambda_{m} \sum_{k=1}^{J_{m}}\left(v, \Phi_{k}\right)_{2}\left\langle\Phi_{k}, w\right\rangle_{2},
$$

and so (70) is equal to $\langle f, w\rangle_{2}$, where

$$
f=\lambda_{m} \mathcal{R}_{1}+\lambda_{m}^{2} Q_{m} B \varphi-\lambda_{m}^{2} \sum_{k=1}^{J_{m}}\left(\mathcal{R}_{1}+\lambda_{m} B \varphi, \Phi_{k}\right){ }_{2} \Phi_{k} .
$$

It is clear that

$$
\|f\|_{L^{2}\left(\Omega_{2}\right)} \leq c\left(\left\|\mathcal{R}_{1}\right\|_{2}+\|B \varphi\|_{2}\right) \leq c d^{1 / 2}\|\varphi\|_{1},
$$

where estimates (45) and (70) are used. Now, applying Lemma 3 to equation (69), we get the inequality

$$
\left\|\mathcal{R}_{1}\right\|_{W_{\beta}^{1,2}\left(\Omega_{2}\right)} \leq c d^{1 / 2}\|\varphi\|_{1}
$$

for $|\beta| \leq \beta_{0}$. 

$\psi$ :

Lemma 1 yields the following estimate of expression (47) with $\varphi$ replaced by

$$
\begin{aligned}
& \left|(B \psi, w)_{2}\right| \leq C\left(\left\|\psi_{0}\right\|_{L^{2}\left(\Omega_{1}\right)}\left\|w_{1}\right\|_{L^{2}\left(\Omega_{2}\right)}+\left\|S \psi_{1}\right\|_{L^{2}\left(\Omega_{1}\right)}\|w\|_{L^{2}\left(\Omega_{2}\right)}\right. \\
& \left.+\left\|\psi_{1}\right\|_{L^{2}\left(\Omega_{1}\right)}\left\|w_{0}\right\|_{L^{2}\left(\Omega_{2}\right)}+\|\psi\|_{L^{2}\left(\Omega_{1}\right)}\left\|S^{*} w_{1}\right\|_{L^{2}\left(\Omega_{2}\right)}\right) .
\end{aligned}
$$

Applying Corollary 1 (i) to the terms containing $w_{1}, \psi_{1}, S \psi_{1}$ and $S^{*} w_{1}$, we obtain

$$
\begin{aligned}
& \left|(B \psi, w)_{2}\right| \leq C d^{(1+\beta) / 2}\left(\left\|\psi_{0}\right\|_{L^{2}\left(\Omega_{1}\right)}\left\|w_{1}\right\|_{W_{-\beta}^{1,2}\left(\Omega_{2}\right)}+\left\|S \psi_{1}\right\|_{W_{-\beta}^{1,2}\left(\Omega_{1}\right)}\|w\|_{L^{2}\left(\Omega_{2}\right)}\right. \\
& \left.+\left\|\psi_{1}\right\|_{W_{-\beta}^{1,2}\left(\Omega_{1}\right)}\left\|w_{0}\right\|_{L^{2}\left(\Omega_{2}\right)}+\|\psi\|_{L^{2}\left(\Omega_{1}\right)}\left\|S^{*} w_{1}\right\|_{W_{-\beta}^{1,2}\left(\Omega_{2}\right)}\right)
\end{aligned}
$$

for $|\beta| \leq \beta_{0}$. Using Corollary 3 and noting that $w_{0}=P w$ and $\psi_{0}=P \psi$, we arrive at

$$
\left|(B \psi, w)_{2}\right| \leq C d^{(1+\beta) / 2}\left(\|\psi\|_{1}|| w\left\|_{W_{-\beta}^{1,2}\left(\Omega_{2}\right)}+\right\| \psi\left\|_{W_{-\beta}^{1,2}\left(\Omega_{1}\right)}\right\| w \|_{2}\right) .
$$

Since $\Omega_{1}$ is Lipschitz and $\psi \in X_{m}$, we have that $\psi \in W^{1, p}\left(\Omega_{1}\right)$ for some $p>2$ (see, for example, [1] or [5]), and so

$$
\int_{\Omega_{1}} r^{-\beta}|\nabla \psi|^{2} d x \leq C|| \psi \|_{1}^{2}
$$

with some positive $\beta$, which together with (73) leads to the inequalities

$$
\left|\left(\mathcal{R}_{1}(\varphi), B \psi\right)_{2}\right| \leq C d^{1+\beta / 2}\|\varphi\|_{1}\|\psi\|_{1}
$$

and

$$
\left|(B \varphi, S \psi)_{2}\right| \leq c d^{(1+\beta) / 2}|| \varphi\left\|_{1}\right\| \psi \|_{1}
$$

valid for $\varphi, \psi \in X_{m}$. This implies, in particular, that $\rho_{1}$ can be taken in the form $c d^{(1+\beta) / 2}$ and that the asymptotic formula (35) can be written as follows:

$$
\frac{1}{\mu_{j}}=\frac{1}{\lambda_{m}}+q_{j}+O\left(d^{1+\beta / 2}\right),
$$

where $\left\{q_{j}\right\}_{j=1}^{J_{m}}$ are eigenvalues of the problem

$$
(B \varphi, S \psi)_{2}+\lambda_{m}\left(Q_{m} B \varphi, B \psi\right)_{2}=q(S \varphi, S \psi)_{2} \quad \text { for all } \psi \in X_{m} .
$$

Furthermore, using representation (50) for $\phi \in X_{m}$ and Lemma 2(ii), we have

$$
\|S \varphi-\varphi\|_{2}^{2}=\|\Phi\|_{2}^{2} \leq c d\|\varphi\|_{1}^{2} .
$$

This and the second inequality in (58) show that

$$
\left|(S \varphi, S \psi)_{2}-(\varphi, \psi)_{1}\right| \leq c d\|\varphi\|_{1}\|\psi\|_{1} \quad \text { for } \varphi, \psi \in X_{m} .
$$


Moreover, it follows from (76) and (45) that $q_{j}=O\left(d^{(1+\beta) / 2}\right)$. Now using (71), we obtain

$$
\left(P_{m} B \varphi, B \psi\right)_{2}=\sum_{j=1}^{J_{m}}\left(B \varphi, S \varphi_{j}\right)_{2}\left(S \varphi_{j}, B \psi\right)_{2},
$$

where $S \varphi_{j}=\Phi_{j}, \varphi_{j} \in X_{m}$. Furthermore, applying (76), we get

$$
\left(P_{m} B \varphi, B \psi\right)_{2} \leq c d^{1+\beta}\|\varphi\|_{1}\|\psi\|_{1} .
$$

These observations lead to the following simplification of formula (77)

$$
\frac{1}{\mu_{j}}=\frac{1}{\lambda_{m}}+\tau_{j}+O\left(d^{1+\beta / 2}\right),
$$

where $\left\{\tau_{j}\right\}_{j=1}^{J_{m}}$ are eigenvalues of the problem

$$
(B \varphi, S \psi)_{2}+\lambda_{m}(B \varphi, B \psi)_{2}=\tau(\varphi, \psi)_{1} \text { for all } \psi \in X_{m} .
$$

In order to simplify the expression $(B \varphi, B \psi)_{2}$, we write

$$
K_{2} \varphi=\frac{1}{\lambda_{m}} S \varphi-\frac{1}{\lambda_{m}} \Psi
$$

where $\Psi=\Psi_{\varphi} \in H_{2}$. Using the definitions of the operators $K_{2}$ and $S$, one can verify that $\Psi$ solves the problem (6). Clearly, $\Psi$ is orthogonal to $H_{1} \cap H_{2}$.

According to (50) and (81), we have $B \varphi=K_{2} \Phi_{\varphi}-\lambda_{m}^{-1} \Psi_{\varphi}$, and so

$$
(B \varphi, B \psi)_{2}=\frac{1}{\lambda_{m}^{2}}\left(\Psi_{\varphi}, \Psi_{\psi}\right)_{2}+\left(K_{2} \Phi_{\varphi}, K_{2} \Phi_{\psi}\right)_{2}-\frac{1}{\lambda_{m}}\left(\left(K_{2} \Phi_{\varphi}, \Psi_{\psi}\right)_{2}+\left(\Psi_{\varphi}, K_{2} \Phi_{\psi}\right)_{2}\right) .
$$

Using the third inequality (58) combined with the definition of $\Psi$, we get

$$
(\Psi, \Psi)_{2} \leq c\left(\int_{\Gamma_{1}}|\nabla \varphi|^{2} d \Gamma\right)^{1 / 2}\left(\int_{\Gamma_{1}}|\Psi|^{2} d x\right)^{1 / 2} \leq c\left(\int_{\Gamma_{1}}|\Psi|^{2} d x\right)^{1 / 2} .
$$

Reasoning as in the proof of Lemma 1, we derive the estimate

$$
\int_{\Gamma_{1}}|\Psi|^{2} d x \leq c d \int_{D_{d}^{+} \backslash D_{d}^{-}}|\nabla \Psi|^{2},
$$

cf. (56). Estimating the right-hand side in (83) by the right-hand side in the last inequality, we obtain

$$
(\Psi, \Psi)_{2} \leq c d,
$$

which together with (55) and (56) yields

$$
\int_{D_{d}^{+} \backslash D_{d}^{-}}|\Psi|^{2} d x \leq c d^{2} \int_{D_{d}^{+} \backslash D_{d}^{-}}|\nabla \Psi|^{2} d x
$$


and

$$
\int_{\partial D_{d}^{-}}|\Psi|^{2} d \Gamma \leq c d \int_{D_{d}^{+} \backslash D_{d}^{-}}|\nabla \Psi|^{2} d x .
$$

Applying again [5, Theorem 2.1] to the function $\Psi$ in $D_{d}^{-}$, we obtain

$$
\int_{D_{d}^{-}}|\Psi|^{2} d \Gamma \leq c d \int_{D_{d}^{+} \backslash D_{d}^{-}}|\nabla \Psi|^{2} d x,
$$

which implies that

$$
\int_{D_{d}^{-}}|\Psi|^{2} d x=O\left(d^{2}\right),
$$

and so (84) yields

$$
\int_{\Omega_{2}}|\Psi|^{2} d x \leq C d^{2} .
$$

The last estimate together with (50) and (82) leads to

$$
(B \varphi, B \psi)_{2}=\frac{1}{\lambda_{m}^{2}}\left(\Psi_{\varphi}, \Psi_{\psi}\right)_{2}+O\left(d^{2}\right),
$$

which along with (80) and (62) gives (5).

Thus we the proof of Theorem 1 is complete.

We conclude this section by the following representation:

$$
\Psi_{\varphi}=\varphi-T_{\varphi},
$$

where $T=T_{\varphi} \in H_{2}$ solves the problem $(T, w)_{2}=\lambda_{m}\langle\varphi, w\rangle_{2}$ for all $w \in H_{2}$.

\subsection{The case when $\Gamma_{1}$ is $C^{1}$-smooth. Proof of Theorem 2}

In this section, we suppose that $\Gamma_{1}$ belongs to the class $C^{1}$, and the operator $\mathcal{A}$ satisfies (8).

Here we use a regularized distance $\rho=\rho(x)$ to the boundary $\partial D_{2 d}^{+}$. One can define $\rho$ so that it is smooth in $D_{2 d}^{+}, c_{1} r \leq \rho \leq c_{2} r$ and $\left|\partial^{\alpha} \rho\right| \leq c_{\alpha} r^{1-|\alpha|}$. Since $\Gamma_{1}$ is $C^{1}$-smooth, $\rho$ can be chosen that it also satisfies the estimate

$$
\left|\partial_{x_{j}} \partial_{x_{i}} \rho\right| \leq \varepsilon \rho^{-1}+C_{\varepsilon} \rho,
$$

where $\varepsilon$ is an arbitrary positive number. Let us explain how such a function can constructed locally. Consider the Lipschitz graph domain

$$
G=\left\{x=\left(x^{\prime}, x_{n}\right) \in \mathbb{R}^{n}: x_{n}>h\left(x^{\prime}\right)\right\},
$$

where $h$ is a Lipschitz function. Let us extend $h$ onto $\mathbb{R}_{+}^{n}$ as follows:

$$
\Phi(\xi)=\int_{\mathbb{R}^{n-1}} \omega_{\mu}(\tau) h\left(\tau \xi_{n}+\xi^{\prime}\right) d \tau,
$$


where $\omega_{\mu}(\tau)=\mu^{1-n} \omega(\tau / \mu)$ and $\omega(\tau)=c \exp \left(\left(|\tau|^{2}-1\right)^{-1}\right)$ for $|\tau| \leq 1$, and $\omega(\tau)=0$ for $|\tau|>1$. The constant $c$ is chosen so that

$$
\int_{\mathbb{R}^{n-1}} \omega(\tau) d \tau=1
$$

It is clear that $\Phi$ is continuous on $\overline{\mathbb{R}_{+}^{n}}$ and $\Phi\left(\xi^{\prime}, 0\right)=h\left(\xi^{\prime}\right)$. It was shown in [9] that the mapping $x^{\prime}=\xi^{\prime}, \quad x_{n}=\xi_{n}+\Phi(\xi)$ is a bi-Lipschitz isomorphism of $\mathbb{R}_{+}^{n}$ onto $G$, provided $\mu$ in (88) is sufficiently small. Taking, for example, $\rho(x)=\xi_{n}(x)$, one verifies directly that all required properties are fulfilled.

Lemma 4 Let $v \in \stackrel{\circ}{W}^{1,2}\left(D_{d}^{+}\right)$and let the operator $\mathcal{A}$ satisfy (8). Then for every $\varepsilon>0$ there exists a constant $C_{\varepsilon}$ such that

$$
J \leq \frac{4}{(1-\varepsilon)^{2}}(v, v)_{D_{d}^{+}}+C_{\varepsilon} \int_{D_{d}^{+}}|v|^{2} d x,
$$

where

$$
J=\int_{\Omega} \rho^{-2} \sum_{i, j=1}^{n}\left(A_{i j} \partial_{i} \rho \partial_{j} \rho v, v\right) d x
$$

Proof. We have that

$$
\begin{aligned}
& J=-\int_{\Omega} \sum_{i, j=1}^{n}\left(A_{i j} \partial_{i}\left(\rho^{-1}\right) \partial_{j} \rho v, v\right) d x \\
& =\int_{\Omega} \rho^{-1} \sum_{i, j=1}^{n}\left(\left(A_{i j} \partial_{i} v, \partial_{j} \rho v\right)+\left(A_{i j} \partial_{j} \rho v, \partial_{i} v\right)+\left(A_{i j}\left(\partial_{i} \partial_{j} \rho\right) v, v\right)\right) d x
\end{aligned}
$$

which together with (8) and (87) implies

$$
J \leq 2(v, v)_{D_{d}^{+}}^{1 / 2} J^{1 / 2}+\varepsilon J+C_{\varepsilon} \int_{D_{d}^{+}} \rho^{-1}|v|^{2} d x .
$$

The last inequality yields (89).

Lemma 5 Assume that the boundary $\Gamma_{1}$ is of class $C^{1}$ and that the operator $\mathcal{A}$ satisfies (8). Then for $\beta \in(-1,1)$ the following assertions are valid:

(i) estimate (64) holds with a positive constant $C$ which may depend on $\beta$;

(ii) all assertions of Corollary 3 hold.

Proof. Putting $v=\rho^{\beta / 2} w$, we have that

$$
\left(w, \rho^{\beta} w\right)_{\Omega}=(v, v)_{\Omega}-\frac{\beta^{2}}{4} J
$$

where $J$ is given by (90). Then (89) yields

$$
\left(w, \rho^{\beta} w\right)_{\Omega} \geq\left(1-\frac{\beta^{2}}{(1-\varepsilon)^{2}}\right)(v, v)_{\Omega}-C_{\varepsilon} \int_{\Omega}|v|^{2} d x .
$$


Using this inequality with $|\beta| \leq 1-\varepsilon$ for estimating the second term in (65), we obtain

$$
\begin{aligned}
& (v, v)_{\Omega} \leq C_{\varepsilon}\left(\sum_{i=1}^{n}\left\langle r^{\beta / 2} f_{k}, \partial_{k} v\right\rangle_{\Omega}+\frac{\beta}{2} \sum_{i=1}^{n}\left\langle r^{\beta / 2} f_{k}, v r^{-1} \partial_{k} r\right\rangle_{\Omega}\right. \\
& \left.+\left\langle r^{\beta / 2+1} f_{0}, r^{-1} v\right\rangle_{\Omega}+\int_{\Omega}|v|^{2} d x\right),
\end{aligned}
$$

which together with the Hardy inequality (54) gives

$$
(v, v)_{\Omega} \leq C_{\varepsilon} \int_{\Omega}\left(r^{\beta} \sum_{k=1}^{n}\left|f_{k}\right|^{2}+r^{\beta+2}\left|f_{0}\right|^{2}\right) d x+C_{\varepsilon}(u, u)_{\Omega} .
$$

Estimating $(u, u)_{\Omega}$ with the help of (64), where $\beta=0$, we get (64) with $\beta \leq 0$.

Now, let $\beta \in(0,1)$. In $(63)$, we choose a solution of the problem

$$
(w, V)_{2}=\langle u, V\rangle_{2} \quad \text { for all } V \in H
$$

as $w$. Then (63) takes the form

$$
\langle u, u\rangle_{2}=\sum_{k=1}^{n}\left\langle f_{k}, \partial_{k} w\right\rangle_{2}+\left\langle f_{0}, w\right\rangle_{2} .
$$

Furthermore, applying (64) with negative $\beta$ to $w$, we obtain

$$
\int_{\Omega}\left(r^{-\beta}|\nabla w|^{2}+r^{-\beta-2}|\nabla w|^{2}\right) \leq C_{\beta} \int_{\Omega} r^{-\beta+2}|u|^{2} d x .
$$

Therefore, we have

$$
\begin{aligned}
& \langle u, u\rangle_{2} \leq \sum_{k=1}^{n}\left(\int_{\Omega} r^{\beta}\left|f_{k}\right|^{2} d x\right)^{1 / 2}\left(\int_{\Omega} r^{-\beta}|\nabla w|^{2} d x\right)^{1 / 2} \\
& +\left(\int_{\Omega} r^{2+\beta}\left|f_{0}\right|^{2} d x\right)^{1 / 2}\left(\int_{\Omega} r^{-2-\beta}|w|^{2} d x\right)^{1 / 2}
\end{aligned}
$$

which implies

$$
\langle u, u\rangle_{2} \leq C\left(\sum_{k=1}^{n} \int_{\Omega} r^{\beta}\left|f_{k}\right|^{2} d x+\int_{\Omega} r^{2+\beta}\left|f_{0}\right|^{2} d x\right)^{1 / 2}\left(\int_{\Omega} r^{-\beta+2}|u|^{2} d x\right)^{1 / 2} .
$$

Since $2-\beta>0$, the last inequality yields that

$$
\langle u, u\rangle_{2} \leq C \sum_{k=1}^{n} \int_{\Omega} r^{\beta}\left|f_{k}\right|^{2} d x+\int_{\Omega} r^{2+\beta}\left|f_{0}\right|^{2} d x .
$$

Finally, using this inequality for estimating of the last term in (93) we get (64) for $\beta \in(0,1)$. This completes the proof of assertion (i).

Assertion (ii) follows from (i) in the same way as Corollary 3 follows from Lemma 3.

In the next lemma weighted estimates for $B w$ are obtained. 
Lemma 6 Let $\beta \in(0,1)$, then we have

$$
\int_{\Omega_{2}} r^{\beta}|\nabla B \varphi|^{2} d x \leq C_{\beta} d^{2 \beta}\|\varphi\|_{1}^{2}
$$

for $\varphi \in X_{m}$.

Proof. We apply the representation $S^{*} w=\zeta_{d}(x) w+W$, where $\zeta_{d}=1$ in $D_{2 d}^{-}$ and $\zeta_{d}=0$ outside $D_{d}^{-}$. The function $\zeta_{d}$ can be chosen so that $\left|\partial_{x}^{\alpha} \zeta_{d}\right| \leq c_{\alpha} r^{-|\alpha|}$. Then the function $W$ satisfies the equation

$$
(W, v)_{1}=\left(\left(1-\zeta_{d}\right) w, v\right)_{1}=\sum_{i, j=1}^{n} \int_{\Omega_{1}}\left(A_{i j}\left(\left(1-\zeta_{d}\right) \partial_{i} w-\partial_{i} \zeta_{i} w, \partial_{j} v\right)\right) d x
$$

In view of Lemma $5(\mathrm{i})$, we get

$$
\int_{\Omega_{1}} r^{\beta}|\nabla W|^{2} d x \leq C \int_{\Omega_{1}} r^{\beta}\left(\left|1-\zeta_{d}\right|^{2}|\nabla w|^{2}+\left|\nabla \zeta_{d}\right|^{2}|w|^{2}\right) d x .
$$

Using (55), we obtain

$$
\int_{\Omega_{1}} r^{\beta}|\nabla W|^{2} d x \leq C \int_{\Omega_{1} \backslash D_{2 d}^{-}} r^{\beta}|\nabla w|^{2} d x .
$$

It follows from (46) that

$$
(B \varphi, w)_{2}=\left\langle\Phi_{\varphi}, w\right\rangle_{2}-\left\langle\varphi,\left(\zeta_{d}-1\right) w+W\right\rangle_{1}
$$

Since $\varphi \in X_{m}$, we have $(\varphi, W)_{1}=\lambda_{m}\langle\varphi, W\rangle_{1}$, and so

$$
(B \varphi, w)_{2}=\left\langle\Phi_{\varphi}, w\right\rangle_{2}-\left\langle\varphi,\left(\zeta_{d}-1\right) w\right\rangle_{1}-\lambda_{m}^{-1}\left(\varphi,\left(1-\zeta_{d}\right) w\right)_{1} .
$$

Using again Lemma 5(i) together with (55), we get

$$
\int_{\Omega_{2}} r^{\beta}|\nabla(B \varphi)|^{2} d x \leq C_{\beta}\left(\int_{\Omega_{2} \backslash D_{2 d}^{-}} r^{\beta}|\nabla \varphi|^{2} d x+\int_{\Omega_{2}} r^{2+\beta}\left|\Phi_{\varphi}\right|^{2} d x\right) .
$$

This inequality and Lemma 2(ii) give

$$
\int_{\Omega_{2}} r^{\beta}|\nabla B \varphi|^{2} d x \leq C_{\beta}\left(\int_{\Omega_{2} \backslash D_{2 d}^{-}} r^{\beta}|\nabla \varphi|^{2} d x+d^{2}\|\varphi\|_{1}^{2}\right) .
$$

It follows from (95) that

$$
\int_{\Omega_{2}} r^{\beta}|\nabla B \varphi|^{2} d x \leq C_{\beta} d^{2 \beta}\left(\int_{\Omega_{2} \backslash D_{2 d}^{-}} r^{-\beta}|\nabla \varphi|^{2} d x+\|\varphi\|_{1}^{2}\right)
$$

for $\beta \in(0,1)$. Since the function $\varphi$ belongs to $W^{1, p}(\Omega)$ for all $p>1$, the integral in the left-hand side is bounded by $\|\varphi\|_{1}^{2}$ and we obtain (94). The proof is complete. 
Let us consider the right-hand side $g$ in (66). We have

$$
(g, w)_{2}=\lambda_{m}\left\langle Q_{m} B \varphi, Q_{m} w\right\rangle_{2}=\lambda_{m}\left\langle B \varphi, Q_{m} w\right\rangle_{2}-\lambda_{m}\left\langle P_{m} B \varphi, Q_{m} w\right\rangle_{2} .
$$

Using the Hardy inequality (54), we estimate the first term in the right-hand side as follows:

$$
\begin{aligned}
& \left|\lambda_{m}\left\langle B \varphi, Q_{m} w\right\rangle_{2}\right| \leq \lambda_{m}\|B \varphi\|_{L^{2}\left(\Omega_{2}\right)}\left\|Q_{m} w\right\|_{L^{2}\left(\Omega_{2}\right)} \\
& \leq C_{\beta}\left(\int_{\Omega_{2}} r^{\beta}|\nabla B \varphi|^{2} d x\right)^{1 / 2}\left\|Q_{m} w\right\|_{2},
\end{aligned}
$$

where $\beta \in(0,1)$. Applying (94), we get

$$
\left|\lambda_{m}\left\langle B \varphi, Q_{m} w\right\rangle_{2}\right| \leq C_{\beta} d^{\beta}\|\varphi\|_{1}\|w\|_{2} .
$$

For estimating the second term in the right-hand side of (97), we note that according to (71) with $\Phi_{k}=S \varphi_{k}, \varphi_{k} \in X_{m}$, we have

$$
\left\langle P_{m} B \varphi, Q_{m} w\right\rangle_{2}=\sum_{k=1}^{J_{m}}\left(B \varphi, S \varphi_{k}\right)_{2}\left\langle\Phi_{k}, Q_{m} w\right\rangle_{2} .
$$

Using estimate (76), in which the exponent $\beta$ can be taken arbitrary close to 1 because we deal with the $C^{1}$-case, we obtain

$$
\left|\left\langle P_{m} B \varphi, Q_{m} w\right\rangle_{2}\right| \leq C_{\beta} d^{(1+\beta) / 2}\|\varphi\|_{1}\|w\|_{2} .
$$

Applying inequalities (98) and (99) for estimating the right-hand side in (97), we arrive at

$$
\left|(g, w)_{2}\right| \leq C_{\beta} d^{\beta}|| \varphi\left\|_{1}\right\| w \|_{2} .
$$

This leads to the estimate

$$
\|g\|_{2} \leq C_{\beta} d^{\beta}\|\varphi\|_{1},
$$

which combined with $(67)$ gives

$$
\left\|\mathcal{R}_{1}\right\|_{2} \leq C_{\beta} d^{\beta}\|\varphi\|_{1} .
$$

Now let us turn again to equation (69), in which the right-hand side can be written as $\langle f, w\rangle_{2}$ with $f$ given by (72). Using Lemma 5, we obtain

$$
\int_{\Omega_{2}} r^{-\beta}\left|\nabla \mathcal{R}_{1}\right|^{2} d x \leq C \int_{\Omega_{2}} r^{2-\beta}|f|^{2} d x,
$$

where $\beta \in(0,1)$. One can check that

$$
\int_{\Omega_{2}} r^{2-\beta}|f|^{2} d x \leq C \int_{\Omega_{2}} r^{\beta}\left(\left|\nabla \mathcal{R}_{1}\right|^{2}+|\nabla B \varphi|^{2}\right) d x .
$$


By (100) and (94), the right-hand side does not exceed $c d^{2 \beta}\|\varphi\|_{1}$. Therefore,

$$
\int_{\Omega_{2}} r^{-\beta}\left|\nabla \mathcal{R}_{1}\right|^{2} d x \leq C d^{\beta}\|\varphi\|_{1} .
$$

Applying this for estimating the right-hand side in (74), we get

$$
\left|\left(\mathcal{R}_{1}(\varphi), B \psi\right)_{2}\right| \leq C d^{(1+3 \beta) / 2}\|\varphi\|_{1}\|\psi\|_{1} .
$$

This leads to

$$
\frac{1}{\mu_{j}}=\frac{1}{\lambda_{m}}+q_{j}+O\left(d^{2 \beta}\right),
$$

where $\left\{q_{j}\right\}_{j=1}^{J_{m}}$ are eigenvalues of problem (78). Repeating the reasoning in Sect. 3.3 that goes after formula (78), we obtain

$$
\frac{1}{\mu_{j}}=\frac{1}{\lambda_{m}}+\tau_{j}+O\left(d^{2 \beta}\right),
$$

where $\left\{\tau_{j}\right\}_{j=1}^{J_{m}}$ are eigenvalues of problem (80).

Thus, Theorem 2 is proved.

\subsection{On the validity of the Hadamard formula. Proof of Theorem 3.}

We assume that $\Omega_{1}$ is of class $C^{1, \alpha}, \alpha \in(0,1)$. This implies, in particular, that the function $\varphi$ belongs to $C^{1, \alpha}\left(\Omega_{1}\right)^{M}$. We will use the following coordinates in a neighborhood of $\Gamma_{1}$ : if $x$ belongs to this neighborhood, then $z \in \Gamma_{1}$ is the nearest to $x$ point on $\Gamma_{1}$ and $\tau$ is such that $x=z+\tau \nu(z)$, where $\nu(z)$ is the unit outward normal to $\Gamma_{1}$ at $z$. Then $\Gamma_{2}$ is given by $\tau=h(z)$. We suppose that $\left|\nabla_{z} h(z)\right|$ is bounded by a constant independent of $d$, and $h$ satisfies (9). Our immediate aim is to prove formula (10) with $\theta_{j}$ given by (11).

Let $\Omega_{d}$ be the domain whose boundary $\Gamma_{d}$ is given by $\tau=d$. We split $T_{\varphi}$ into the sum $T_{\varphi}^{(1)}+T_{\varphi}^{(2)}$, where $T_{\varphi}^{(1)}$ solves the problem $-\mathcal{A} T_{\varphi}^{(1)}=\lambda_{m} \varphi$ in $\Omega_{d}$ and $T_{\varphi}^{(1)}=0$ on $\Gamma_{d}$. The function $T_{\varphi}^{(2)}$ is a solution of the following problem: $\mathcal{A} T_{\varphi}^{(2)}=0$ in $\Omega_{2}$ and $T_{\varphi}^{(2)}=-T_{\varphi}^{(1)}$ on $\Gamma_{2}$.

Since $T_{\varphi}^{(1)} \in C^{1, \alpha}\left(\Omega_{d}\right)^{M}$ and $T_{\varphi}^{(1)}=0$ on $\Gamma_{d}$, we obtain that

$$
\left|T_{\varphi}^{(1)}(x)\right| \leq c|h(z)-d| \text { and }\left|\nabla_{z} T_{\varphi}^{(1)}(x)\right| \leq c|h(z)-d|^{\alpha} \text { on } \Gamma_{2} .
$$

This together with (9) implies

$$
\int_{\Gamma_{2}}\left|T_{\varphi}^{(1)}\right|^{2} d z=O\left(d^{2}\right) \text { and } \int_{\Gamma_{2}}\left|\nabla_{z} T_{\varphi}^{(1)}(z, h(z))\right|^{2} d z=o(1) .
$$

Applying Theorems 2.1 and 4.2 from [5] to the solution $T_{\varphi}^{(2)}$ in $\Omega_{2}$, we obtain

$$
\int_{\Gamma_{2}}\left|T_{\varphi}^{(2)}\right|^{2} d \Gamma \leq c \int_{\Gamma_{2}}\left|T_{\varphi}^{(1)}(z)\right|^{2} d \Gamma=O\left(d^{2}\right)
$$


and

$$
\int_{\Gamma_{2}}\left|\nabla T_{\varphi}^{(2)}\right|^{2} d \Gamma \leq c \int_{\Gamma_{2}}\left|\nabla_{z} T_{\varphi}^{(1)}(z, h(z))\right|^{2} d \Gamma=o(1) .
$$

Hence we have that

$$
\left(T_{\varphi}^{(2)}, T_{\psi}^{(2)}\right)_{\Omega_{2}}=\int_{\Gamma_{2}}\left(T_{\varphi}^{(2)}, N T_{\psi}^{(2)}\right) d x=o(d)
$$

and so

$$
\left(\Psi_{\varphi}, \Psi_{\psi}\right)_{2}=\left(\varphi-T_{\varphi}^{(1)}, \psi-T_{\psi}^{(1)}\right)_{2}+o(d)
$$

Let us consider the function $V=\varphi-T_{\varphi}^{(1)}$ in $\Omega_{1} \cap \Omega_{2}$. This function satisfies $\mathcal{A} V=0$ in $\Omega_{1} \cap \Omega_{2}$ and $V=\varphi-T_{\varphi}^{(1)}$ on the boundary of $\Omega_{1} \cap \Omega_{2}$, say, $\Gamma_{12}$. Since $\Gamma_{12}$ is given by $\tau=h_{1}(z), h_{1}(z)=\min (h(z), 0)$, we obtain

$$
\begin{aligned}
& \int_{\Gamma_{12}}\left|\nabla_{z} T_{\varphi}^{(1)}\left(z, h_{1}(z)\right)\right|^{2} d \Gamma \\
& \leq \int_{\Gamma_{12}}\left(\left|T_{\varphi, z}^{(1)}\left(z, h_{1}(z)\right)\right|+\left|T_{\varphi, \tau}^{(1)}\left(z, h_{1}(z)\right)\right|\left|\nabla_{z} h_{1}(z)\right|\right)^{2} d \Gamma=o(1)
\end{aligned}
$$

where relations (9) and (101) are taken into account, and $T_{\varphi, z}^{(1)}$ and $T_{\varphi, \tau}^{(1)}$ stand for the derivatives with respect to $z$ and $\tau$, respectively. Furthermore, we have

$$
|\varphi(x)| \leq c|\tau|, \quad\left|\nabla_{z} \varphi(x)\right| \leq c|\tau|^{\alpha} \quad \text { and } \quad\left|\partial_{\tau} \varphi(x)\right| \leq c
$$

because $\varphi \in C^{1, \alpha}\left(\Omega_{1}\right)^{M}$. This leads to the following relations:

$$
\int_{\Gamma_{12}}|\varphi|^{2} d z=O\left(d^{2}\right) \text { and } \int_{\Gamma_{12}}\left|\nabla_{z} \varphi(z, h(z))\right|^{2} d z=o(1) .
$$

Using the first inequalities (101) and (103) combined with [5, Theorem 2.1], we obtain

$$
\int_{\Gamma_{12}}|V|^{2} d \Gamma \leq c \int_{\Gamma_{12}}\left|\varphi-T_{\varphi}^{(1)}\right|^{2} d \Gamma=O\left(d^{2}\right) .
$$

In view of (102), (104) and (9), we apply [5, Theorem 4.2], thus getting

$$
\int_{\Gamma_{12}}|\nabla V|^{2} d \Gamma=o(1)
$$

and so

$$
(V, V)_{\Omega_{1} \cap \Omega_{2}}=\int_{\Gamma_{12}}(V, N V) d x=o(d) .
$$

This and (86) lead to

$$
\left(\Psi_{\varphi}, \Psi_{\varphi}\right)_{2}=\left(T_{\varphi}^{(1)}(\varphi), T_{\psi}^{(1)}(\psi)\right)_{\Omega_{2} \backslash \Omega_{1}}+o(d) .
$$

Since $T_{\varphi}^{(1)} \in C^{1, \alpha}$, we obtain

$$
\left|\nabla T_{\varphi}^{(1)}(z, \tau)-\nabla T_{\varphi}^{(1)}(z, 0)\right| \leq c|\tau|^{\alpha} .
$$


This and (105) imply

$$
\left.{ }^{\left(T_{\varphi}^{(1)}\right.}, T_{\psi}^{(1)}\right)_{\Omega_{2} \backslash \Omega_{1}}=\int_{\Gamma_{0}} h(z)\left(A_{i j} \nu_{j} \nu_{i} \partial_{\nu} \varphi, \partial_{\nu} \psi\right) d z+O\left(d^{1+\alpha}\right),
$$

where $\Gamma_{0}=\Gamma_{1} \cap \Omega_{2}$.

Using (103) and (9), we obtain

$$
\int_{\Gamma_{2}}|\varphi(z+h(z) \nu)|^{2} d z=O\left(d^{2}\right) \text { and } \int_{\Gamma_{2}}\left|\nabla_{z} \varphi(z+h(z) \nu)\right|^{2} d z=o(1) .
$$

Applying Theorems 4.2 and Theorem 2.1 [5] to the problem for $\Phi_{\varphi}$, we obtain

$$
\int_{\Gamma_{2}}|\nabla \Phi|^{2} d \Gamma=0(1) \text { and } \int_{\Gamma_{2}}|\Phi|^{2} d \Gamma=O\left(d^{2}\right)
$$

which leads to

$$
\left(\Phi_{\varphi}, \Phi_{\psi}\right)_{2}=\int_{\Gamma_{2}}\left(\Phi_{\varphi}, N \Phi_{\psi}\right) d x=o(d) .
$$

Reasoning in the same way as in the case of the function $T_{\varphi}^{(1)}$, we get

$$
(\varphi, \psi)_{\Omega_{1} \backslash \Omega_{2}}=\int_{\Gamma_{1} \backslash \Gamma_{0}}|h(z)|\left(A_{i j} \nu_{j} \nu_{i} \partial_{\nu} \varphi, \partial_{\nu} \psi\right) d z+o(d) .
$$

Finally, let us consider the terms $\left(\Psi_{\varphi}, \psi\right)_{2}$ and $\left(\varphi, \Psi_{\psi}\right)_{2}$. Denote by $P_{12}$ the orthogonal projector onto $H_{1} \cap H_{2}$ and represent $\psi$ as $P_{12} \psi-\Phi_{\psi}^{*}$. Then $\Phi_{\psi}^{*}$ is harmonic in $\Omega_{1} \cap \Omega_{2}$ and $\Phi_{\psi}^{*}+\psi \in H_{1} \cap H_{2}$. Similar to (108) one derives the estimate $\left(\Phi_{\psi}^{*}, \Phi_{\psi}^{*}\right)_{2}=o(d)$. This relation together with (106) implies

$$
\left(\Psi_{\varphi}, \psi\right)_{2}=o(d)
$$

and similarly $\left(\varphi, \Psi_{\psi}\right)_{2}=o(d)$

Now these formulae together with (108), (109), (106), (107) along with (4), (5) give (10) and (11). Thus, the following theorem is proved.

Theorem 6 Let $\Omega_{1}$ be a domain with $C^{1, \alpha}$ boundary with some $\alpha \in(0,1)$. Let the boundary of $\Omega_{2}$ be given by $\tau=h(z)$, where $h$ is a continuous function such that $h<d$ and condition (9) holds for it. Then the asymptotic formula (10) is valid, in which $\theta_{j}$ are eigenvalues of the spectral problem (11).

\subsection{Plane domains with corner points; proof of (15) and (14)}

In this section, we consider the $2 \mathrm{D}$ case. We assume that the boundary of $\Omega_{1}$ contains corner points $p_{1}, \ldots, p_{l}$, outside of which the boundary belongs to the class $C^{1, \delta}, \delta \in(0,1)$. We denote by $\alpha_{s}$ the interior angle at $p_{s}$. It is clear that $\alpha_{s}<2 \pi$. In view of known results about the behavior of solutions of the 
Dirichlet problem for second order systems near corner points (see [8, Sect. 8.6.3 and 8.7.1]), there exist numbers $\lambda_{s}>1 / 2$ such that

$$
|\varphi(x)|+r|\nabla \varphi(x)|+\frac{\left|r(x)^{1+\delta} \nabla \varphi(x)-r^{1+\delta}(y) \nabla \varphi(y)\right|}{|x-y|^{\delta}} \leq C R,
$$

where $r=r_{1} r_{2} \cdots r_{l}, R=\Pi_{s=1}^{l} r_{s}^{\lambda_{s}}$ and $r_{s}(x)=\left|x-p_{s}\right|$. Here and in what follows, by $c, C, c_{k} \ldots$ we denote various constants independent of $d$.

We assume that $\Omega_{2} \subset \Omega_{1}$, and so the asymptotic formula (4) is valid, but the functions $\Psi_{\varphi}$ and $\Psi_{\psi}$ in (5) are equal to zero in the present case. Therefore, the numbers $\left\{\tau_{j}\right\}_{j=1}^{J_{m}}$ in (4) are eigenvalues of the problem

$$
\frac{1}{\lambda_{m}}\left(-\left(\Phi_{\varphi}, \Phi_{\psi}\right)_{2}-(\varphi, \psi)_{\Omega_{1} \backslash \Omega_{2}}\right)=\tau(\varphi, \psi)_{2} \text { for all } \psi \in X_{m} .
$$

Let $\Gamma^{\prime}=\left\{z \in \Gamma_{1}:\left|z-p_{s}\right|>c d\right\}$. In a neighborhood of $\Gamma^{\prime}$, we use the coordinates $z$ and $\tau$, the first of which is measured along $\Gamma^{\prime}$, whereas $\tau$ is the normal coordinate. The part of $\Gamma_{2}$ lying outside $\Omega_{1}$ is given by $\tau=h(z, d)$. We denote this part by $\Gamma_{2}^{\prime}$, and assume that $h \leq d,\left|\partial_{z} h(z, d)\right| \leq C$ and

$$
\int_{\Gamma^{\prime}}\left|h^{\prime}(z, d)\right|^{2} R^{2} r^{-2} d z=o(1) .
$$

We also suppose that the remaining part of $\Gamma_{2}$ is located in $B\left(p_{s}, c_{2} d\right) \backslash B\left(p_{s}, c_{1} d\right)$, where $B(p, r)$ is the disc having its center at $p$ and radius equal to $r$; let each of these parts be Lipschitz with uniformly bounded Lipschitz constants.

Since $\varphi=0$ on the boundary of $\Omega_{1}$, we use estimates (111) for obtaining

$$
\left|\partial_{z} \varphi(z, \tau)\right| \leq C \tau^{\delta} R r^{-1-\delta},
$$

and so

$$
\left|\partial_{z} \varphi(z, h(z, d))\right| \leq C\left(\tau^{\delta} R r^{-1-\delta}+R r^{-1}\left|h_{z}^{\prime}\right|\right) .
$$

Furthermore, it follows from (111) that

$$
|\varphi(z, h(z, d))| \leq C h(z, d) R r^{-1} \text { and }|(\nabla \varphi)(z, h(z, d))| \leq C R r^{-1},
$$

which imply

$$
\int_{\Gamma^{\prime}}|\varphi(z, h(z, d))|^{2} d \Gamma=O\left(d^{2}\right) .
$$

Assumption (113) together with (114) gives

$$
\int_{\Gamma^{\prime}}\left|\partial_{z} \varphi\right|^{2} d z=o(1) \quad \text { as } d \rightarrow 0
$$

Moreover, it follows from (111) that

$$
\int_{\Gamma_{2} \backslash \Gamma_{2}^{\prime}}|\nabla \varphi|^{2} d \Gamma=C \max _{s} d^{2 \lambda_{s}-1} .
$$


Applying [5, Theorem 4.2] to the function $\Phi$, we obtain

$$
\int_{\Gamma_{2}}\left|\partial_{\nu} \Phi\right|^{2} d \Gamma=o(1)
$$

Using (115) and (116), we arrive at

$$
\int_{\Omega_{2}}|\nabla \Phi(x)|^{2} d x=\int_{\Gamma_{2}} \varphi \partial_{\nu} \Phi d \Gamma=o(d) .
$$

This together with (112) leads to (15), where the numbers $\left\{\theta_{j}\right\}_{j=1}^{J_{m}}$ are eigenvalues of the problem (14). The reader can check the relation $\theta_{j}=O(d)$, for which purpose the above estimates of $\varphi$ must be applied. If one of the angles, say $\alpha_{s}$, is greater than $\pi$, then [8, Theorem 8.6.2] yields that there is an eigenfunction such that the norm of its gradient is estimated from below by $\mathrm{cr}_{s}^{\lambda-1}$ with $\lambda \in(1 / 2,1)$. Then the Hadamard formula (11) will give the inequality $\theta_{j} \geq c d^{2 \lambda-1}$, which shows that the eigenvalue problem (14) is a right replacement of problem (11) for obtaining the asymptotic formula expressing the eigenvalue $\mu_{j}$ in the case of a non-smooth boundary when a corner point of opening greater than $\pi$ is present.

\section{$4 \quad$ Examples}

In this section, we give applications of the general formula (4) to perturbations of the reference domain which involves removing small cuts or small discs.

Let $\Omega_{1}$ be a $2 D$ domain with a smooth boundary $\Gamma_{1}$ whose length is rescaled to unity. In a neighborhood $\mathcal{V}$ of $\Gamma_{1}$, we introduce coordinates $(z, \tau)$ so that $z$ is the arc length measured from a fixed point of $\Gamma_{1}$, whereas $\tau$ is the oriented distance to the boundary $(\tau>0$ outside $\Omega$ ). We put

$$
\Omega_{2}=\Omega \backslash \cup_{j=1}^{N} \Upsilon_{j}^{\varepsilon}, \quad \Upsilon_{j}^{\varepsilon}=\{x \in \Omega \cap \mathcal{V}: z=j \varepsilon, \tau \in[-\varepsilon \ell(z), 0]\},
$$

where $\ell$ is a positive $C^{\infty}(\Gamma)$-function, $\varepsilon=1 / N$ and $N$ is a large positive integer. Clearly the domains $\Omega_{1}$ and $\Omega_{2}$ satisfy Assumption I made in Introduction with $d=c \varepsilon$. Since the reference domain is smooth and $\Omega_{2} \subset \Omega_{1}$ formulae (4) and (5) are valid with $\Psi=0$ and an arbitrary $\delta \in(0,1)$. Let us construct the function $\Phi_{\varphi}=\Phi$ that solves the problem $\mathcal{A}\left(\partial_{x}\right) \Phi=0$ in $\Omega_{2}$ and $\Phi=-\varphi$ on $\Gamma_{2}$. In the variables $\xi=\left(\xi_{1}, \xi_{2}\right)=\left(\varepsilon^{-1} \tau, \varepsilon^{-1} z\right), \Phi$ is sought in the form

$$
\Phi(x)=\chi(x) \varepsilon w_{\varphi}(\xi, z)+\varepsilon R(x, \varepsilon),
$$

where $\chi$ is a smooth function which has a compact support in $\mathcal{V}$ and is equal to 1 in a neighborhood of $\Gamma_{1}$. Let us describe a family of boundary value problems for $w=w_{\varphi}$. Near every point $z_{0} \in \Gamma_{1}$, the operator $\mathcal{A}$ has the following form in variables $\xi$ :

$$
\mathcal{A}\left(\partial_{x}\right)=\varepsilon^{-2} L_{2}\left(z_{0}, \varepsilon \xi, \partial_{\xi}\right)+\varepsilon^{-1} L_{1}\left(z_{0}, \varepsilon \xi, \partial_{\xi}\right)+L_{0}\left(z_{0}, \varepsilon \xi\right),
$$


where $L_{k}\left(z, \xi, \partial_{\xi}\right)$ is a differential operator of $k$ th order; its coefficients are smooth functions of $z$ and $\xi$. In $\Pi_{z}=\Pi \backslash \mathcal{L}_{z}$, where

$$
\Pi=\left\{\xi: \xi_{1}<0, \xi_{2} \in(-1 / 2,1 / 2)\right\} \text { and } \mathcal{L}_{z}=\left\{\xi=\left(\xi_{1}, 0\right): \xi_{1} \in(-\ell(z), 0)\right\},
$$

the term $w$ is described by the boundary value problem that consists of the following equation:

$$
L_{2}\left(z, 0, \partial_{\xi}\right) w(\xi, z)=0, \quad \xi \in \Pi_{z},
$$

and two types of conditions, namely, the periodicity conditions:

$$
w\left(\xi_{1},-1 / 2, z\right)=w\left(\xi_{1}, 1 / 2, z\right), \quad \partial_{\xi_{2}} w\left(\xi_{1},-1 / 2, z\right)=\partial_{\xi_{2}} w\left(\xi_{1}, 1 / 2, z\right), \quad \xi_{1}<0,
$$

and the Dirichlet boundary conditions imposed on a part of the boundary:

$$
\begin{gathered}
w\left(0, \xi_{2}, z\right)=0, \quad \xi_{2} \in(-1 / 2,1 / 2), \\
w\left(\xi_{1}, 0, z\right)=-\xi_{1} \partial_{\nu} \varphi(0, z), \quad \xi_{1}>-\ell(z) .
\end{gathered}
$$

This problem has a unique solution in the class of vector functions such that the following norm is finite:

$$
\left(\int_{\Pi}\left|\nabla_{\xi} u\right|^{2} d \xi+\int_{-1 / 2}^{1 / 2}\left|u\left(\xi_{1}, 0\right)\right|^{2} d \xi_{1}\right)^{1 / 2} .
$$

Moreover, this solution has the following asymptotic representation at the negative infinity:

$$
w(\xi, z)=\mathbf{M}(z)+o\left(\exp \left(\delta \xi_{1}\right)\right), \quad \delta>0, \xi_{1} \rightarrow-\infty,
$$

where $\mathbf{M}(z)$ is a smooth vector-function of $z$, see [13].

In order to estimate the remainder $R$ in (117), we proceed as follows. We begin with rewriting representation (117) in the form:

$$
\begin{aligned}
& \Phi(x)=\hat{\Phi}^{\varepsilon}(x)+\varepsilon R_{1}(x, \varepsilon), \\
& \hat{\Phi}^{\varepsilon}(x)=\left(-\mathcal{X}_{\varepsilon}(x) \varphi(x)+\mathcal{X}_{\varepsilon}(x) \partial_{\nu} \varphi(z, 0) \tau\right)+\chi(x) \varepsilon w(\xi, z) .
\end{aligned}
$$

Here $\mathcal{X}_{\varepsilon}(x)=0$ for $x \in \Omega \backslash \mathcal{V}$ and $\mathcal{X}_{\varepsilon}(x)=\chi_{0}\left(\varepsilon^{-1} \tau\right)$ for $x \in \Omega \cap \mathcal{V}$, whereas $\chi_{0}$ is a smooth function such that $\chi_{0}(t)=1$ for $|t|<2 \ell_{0}$ and $\chi_{0}(t)=0$ for $|t|>3 \ell_{0}$ and $\ell_{0}=\max \ell(s)$. One can check that

$$
R=R_{1}-\mathcal{X}_{\varepsilon}(x) \frac{\varphi(x)-\partial_{\nu} \varphi(z, 0) \tau}{\varepsilon} .
$$

The main reason for introducing the cut-off function $\mathcal{X}_{\varepsilon}$ in the representation of $\Phi$ is to ensure that the remainder $R_{1}$ satisfies the homogeneous Dirichlet boundary condition on $\partial \Omega^{\varepsilon}$. The function $\Phi$ satisfies the following integral identity:

$$
(\Phi, u)_{2}=0 \text { for all } u \in H_{2} \text { and } \Phi+\varphi \in H_{2} .
$$


If $u=R_{1}$ here, then we have that

$$
\left(R_{1}, R_{1}\right)_{2}=\left\langle\mathcal{A}\left(\partial_{x}\right) \hat{\Phi}^{\varepsilon}, R_{1}\right\rangle_{2} .
$$

Let us estimate the norm $\left\|\rho \mathcal{A}\left(\partial_{x}\right) \hat{\Phi}^{\varepsilon}\right\|_{L^{2}\left(\Omega^{\varepsilon}\right)}$, where $\rho$ is the same as in Sect. 3.4. Since

$$
\begin{aligned}
& \mathcal{A}\left(\partial_{x}\right) \hat{\Phi}^{\varepsilon}=-\mathcal{X}_{\varepsilon}(x) \mathcal{A}\left(\partial_{x}\right)\left(\varphi(x)-\partial_{\nu} \varphi(z, 0) \tau\right)-\left[\mathcal{A}, \mathcal{X}_{\varepsilon}\right]\left(\varphi(x)-\partial_{\nu} \varphi(z, 0) \tau\right) \\
& +\varepsilon\left(\chi(x) \mathcal{A}\left(\partial_{x}\right) w+[\mathcal{A}, \chi] w\right)=I^{\varepsilon}(x)
\end{aligned}
$$

it is straightforward to verify that

$$
\left\|\rho I^{\varepsilon}\right\|_{L^{2}\left(\Omega_{2}\right)}=O\left(\varepsilon^{3 / 2}\right) .
$$

Combining this relation and the Hardy inequality (54), we obtain that

$$
\left(R_{1}, R_{1}\right)_{2} \leq c \varepsilon^{3} .
$$

This along with (120) leads to the inequality

$$
(R, R)_{2} \leq c \varepsilon^{2} .
$$

Noting that

$$
\begin{aligned}
& \left(\chi(x) w_{\varphi}(\xi, z), \chi(x) w_{\psi}(\xi, z)\right)_{2} \\
& =\varepsilon^{-1} \int_{\Gamma_{1}} \int_{-1 / 2}^{1 / 2} \int_{-\infty}^{0} \sum\left(A_{i j} \partial_{\xi_{i}} w_{\varphi}(\xi, z), \partial_{\xi_{j}} w_{\psi}(\xi, z)\right) d \xi d z+O(1),
\end{aligned}
$$

we obtain

$$
\frac{1}{\mu_{j}}=\frac{1}{\lambda_{m}}-\varepsilon \frac{\tau_{j}}{\lambda_{m}}+O\left(\varepsilon^{1+\delta}\right),
$$

where $\delta$ is an arbitrary positive number from the interval $(0,1)$ and $\left\{\tau_{j}\right\}_{j=1}^{J_{m}}$ are the eigenvalues of the problem

$$
\int_{\Gamma_{1}} \int_{-1 / 2}^{1 / 2} \int_{-\infty}^{0} \sum\left(A_{i j} \partial_{\xi_{i}} w_{\varphi}(\xi, z), \partial_{\xi_{j}} w_{\psi}(\xi, z)\right) d \xi d z=\tau(\varphi, \psi)_{1} \quad \text { for all } \psi \in X_{m} \text {. }
$$

The "Hadamard term" here is equal to zero, since $\Omega_{1} \backslash \Omega_{2}$ consists of a finite number of cuts.

The second example is as follows. Let $\Omega_{1}$ be the same as above, and let

$$
\Omega_{2}=\Omega \backslash \cup_{j=1}^{N} \mathcal{Q}_{j}^{\varepsilon}, \quad \mathcal{Q}_{j}^{\varepsilon}=\left\{x \in \Omega \cap \mathcal{V}:(z-j \varepsilon)^{2}+(\tau-q(z) \varepsilon)^{2}<\ell^{2}(z) \varepsilon^{2}\right\},
$$

where $q$ and $\ell$ are smooth functions on $\Gamma_{1}$ such that $0<\ell(z)<q(z)$ and $\ell(z)<1 / 2$. In the same way as in the previous example we introduce the variables $\xi$ and put

$$
\Pi_{z}=\left\{\xi: \xi_{1}<0, \xi_{2} \in(-1 / 2,1 / 2) \text { and }\left(\xi_{1}-q(z)\right)^{2}+\xi_{2}^{2}<\ell(z)^{2}\right\} .
$$


Similarly to the previous case, formulae (4) and (5) are valid with $\Psi=0$ and $\delta \in(0,1)$. Let us construct the function $\Phi_{\varphi}=\Phi$. We seek $\Phi$ in the form

$$
\Phi(x)=\chi(x) \varepsilon w(x, z)+\varepsilon R(x, \varepsilon),
$$

where the function $w=w_{\varphi}$ satisfies the boundary value problem that consists of the following equation:

$$
L_{2}\left(z, 0, \partial_{\xi}\right) w(\xi, z)=0, \quad \xi \in \Pi,
$$

and two types of conditions, namely, the periodicity conditions:

$$
w\left(\xi_{1},-1 / 2, z\right)=w\left(\xi_{1}, 1 / 2, z\right), \quad \partial_{2} w\left(\xi_{1},-1 / 2, z\right)=\partial_{2} w\left(\xi_{1}, 1 / 2, z\right), \quad \xi_{1}<0,
$$

and the Dirichlet boundary conditions on a part of the boundary:

$$
\begin{gathered}
w\left(0, \xi_{2}, z\right)=0, \quad \xi_{2} \in(-1 / 2,1 / 2), \\
w(\xi, z)==-\xi_{1} \partial_{\nu} \varphi(0, z), \text { for }\left(\xi_{1}-q(z)\right)^{2}+\xi_{2}^{2}=\ell(z)^{2} .
\end{gathered}
$$

This problem has a unique solution in the class of vector-functions such that the norm (118) is finite. Moreover, this solution admits the asymptotic representation (119) at the negative infinity, where $\mathbf{M}(z)$ depends smoothly on $z$. Reasoning as above, one can show that the remainder in (124) satisfies (122) and the asymptotic formula (123) is valid, where $\delta \in(0,1)$ is arbitrary, whereas $\left\{\tau_{j}\right\}_{j=1}^{J_{m}}$ are the eigenvalues of the problem

$$
\begin{aligned}
& \left(\int_{\Gamma_{1}} \int_{-1 / 2}^{1 / 2} \int_{-\infty}^{0} \sum\left(A_{i j} \partial_{\xi_{i}} w_{\varphi}(\xi, z), \partial_{\xi_{j}} w_{\psi}(\xi, z)\right) d \xi d z\right. \\
& \left.+\sum_{j=1}^{N} \int_{S_{j}} \xi_{1}^{2}\left(A_{\nu} \partial_{\nu} \varphi(j / N, 0), \partial_{\nu} \psi(j / N, 0)\right) d \xi\right)=\tau(\varphi, \psi)_{1} \text { for all } \psi \in X_{m} .
\end{aligned}
$$

Here $A_{\nu}=\sum A_{i j} \nu_{i} \nu_{j}$ and $S_{j}=\left\{\xi:\left(\xi_{1}-q(j / N)\right)^{2}+\xi_{2}^{2}<\ell(j / N)^{2}\right\}$.

Despite the perturbation of the eigenvalue is of the same order as in the Hadamard formula, the coefficient is different here.

Acknowledgements. V. K. was supported by the Swedish Research Council (VR). S. N. acknowledges the financial support from Linköping University.

\section{References}

[1] Agranovich, M. S. Regularity of variational solutions of linear boundary value problems in Lipschitz domains. (Russian) Funktsional. Anal. i Prilozhen. 40 (2006), no. 4, 83-103, 112; translation in Funct. Anal. Appl. 40 (2006), no. $4,313-329$ 
[2] Bucur, Dorin; Buttazzo, Giuseppe, Variational methods in shape optimization problems. Progress in Nonlinear Differential Equations and their Applications, 65. Birkhäuser Boston, Inc., Boston, MA, 2005.

[3] Burenkov, Victor I.; Lamberti, Pier Domenico Spectral stability of Dirichlet second order uniformly elliptic operators. J. Differential Equations 244 (2008), no. 7, 1712-1740.

[4] Delfour, M. C.; Zolésio, J.-P. Shapes and geometries. Analysis, differential calculus, and optimization. Advances in Design and Control, 4. Society for Industrial and Applied Mathematics (SIAM), Philadelphia, PA, 2001.

[5] Gao, Wen Jie Layer potentials and boundary value problems for elliptic systems in Lipschitz domains. J. Funct. Anal. 95 (1991), no. 2, 377-399.

[6] Hadamard, J., Mémoire sur le problème d'analyse relatif à l'équilibre des plaques élastiques encastrées, Euvres, 2, 1968, 515-631.

[7] Henrot, Antoine, Extremum problems for eigenvalues of elliptic operators. Frontiers in Mathematics. Birkhäuser Verlag, Basel, 2006.

[8] Kozlov, V., Maz'ya, V. and Rossmann, J.: Spectral Problems Associated with Corner Singularities of Solutions to Elliptic Equations, Mathematical Surveys and Monographs 85 (2001), Amer. Math. Soc.

[9] Kozlov, Vladimir; Maz'ya, Vladimir Asymptotic formula for solutions to elliptic equations near the Lipschitz boundary. Ann. Mat. Pura Appl. (4) 184 (2005), no. 2, 185-213.

[10] Kozlov, Vladimir, On the Hadamard formula for nonsmooth domains. J. Differential Equations 230 (2006), no. 2, 532-555.

[11] Kozlov, Vladimir. $L^{q}$-perturbations of leading coefficients of elliptic operators: asymptotics of eigenvalues. Abstr. Appl. Anal. 2006, Art. ID 26845, $15 \mathrm{pp}$.

[12] Kawohl, B., Some nonconvex shape optimization problems. Optimal shape design (Tryia, 1998), 7-46, Lecture Notes in Math., 1740, Springer, Berlin, 2000 .

[13] Nazarov S.A. The polynomial property of self-adjoint elliptic boundaryvalue problems and the algebraic description of their attributes // Uspehi mat. nauk. 1999. V. 54, N 5. P. 77-142. (English transl.: Russ. Math. Surveys. 1999. V. 54, N 5. P. 947-1014)

[14] Sokolowski, Jan; Zolésio, Jean-Paul Introduction to shape optimization. Shape sensitivity analysis. Springer Series in Computational Mathematics, 16. Springer-Verlag, Berlin, 1992. 